\title{
Practical guide for safe central venous catheterization and management 2017
}

\author{
Safety Committee of Japanese Society of Anesthesiologists ${ }^{1}$
}

Received: 2 February 2018 / Accepted: 15 October 2019 / Published online: 30 November 2019

(c) Japanese Society of Anesthesiologists 2019

\begin{abstract}
Central venous catheterization is a basic skill applicable in various medical fields. However, because it may occasionally cause lethal complications, we developed this practical guide that will help a novice operator successfully perform central venous catheterization using ultrasound guidance. The focus of this practical guide is patient safety. It details the fundamental knowledge and techniques that are indispensable for performing ultrasound-guided internal jugular vein catheterization (other choices of indwelling catheters, subclavian, axillary, and femoral venous catheter, or peripherally inserted central venous catheter are also described in alternatives).
\end{abstract}

Keywords Medical education · Ultrasound-guided central venous catheterization · Mechanical complication · Catheterrelated infection · Pediatric

\section{Introduction}

Central venous catheterization is an invasive medical procedure applied widely in pediatric and adult patients not only to measure central venous pressure but also to secure a stable route for continuous drug administration. Incorrect insertion procedure can harm patient. Anesthesiologists regularly perform catheterization during medical care; thus, they need to fully understand its safety and risks and be able to do it properly. This guide describes the current state of central venous catheterization and proposes the directions for the procedure. Note that the items in this guideline are described for general principles. Decisions for the procedure on specific cases must be made by the physicians in charge.

\section{Informed consent}

Life-threatening complications can occur from central venous catheterization. To avoid such complications, the advantages and disadvantages of catheterization must be

Safety Committee of Japanese Society of Anesthesiologists anzen@ anesth.or.jp

1 Japanese Society of Anesthesiologists, Kobe KIMEC Center Building 3F, 1-5-2 Minatojima-Minamimachi, Chuo-ku, Kobe 650-0047, Japan considered, including the alternative tool to use in placing a central venous catheter, the use of a peripherally inserted central catheter (PICC) in certain cases, or the use of other substitute methods. Medical institutes, hospitals, and clinical departments should standardize approaches and techniques used to ensure catheterization safety.

Anesthesiologists' participation in central venous catheterization can be divided into two broad categories: first, when they are asked to perform central venous catheterization that is unrelated to surgical anesthesia, such as when a catheter needs to be inserted for a long period for long-term therapy, for example, in anticancer drug therapy for children; and second, when it is part of the series of steps needed for anesthetic management. In the latter cases, the catheter is usually removed after a short period. The risk of infection differs between these two categories [1]. When catheterization is performed for long-lasting therapeutic purposes, the patient's psychological condition and other factors must be considered, unlike catheterization with surgical anesthesia. In either, the procedure should be explained to the patient and his/her family, and informed consent should be obtained beforehand [2,3]. When central venous catheterization needs to be performed in an emergency case, it is preferable to explain the procedure beforehand even just to the family. If the procedure is part of an extreme lifesaving treatment, it should be explained as soon as possible after the procedure 
to inform the patient. If fluoroscopy or other examinations are needed after catheterization, this should also be explained as well.

The explanations should be as comprehensive as possible, covering areas such as why central venous catheterization is needed, complications that could occur, and how unexpected complications would be dealt with. Techniques and complications are described below. If the duration of catheterization becomes longer than expected, an additional explanation should be given and approval should be obtained when replacement becomes necessary.

\section{Educational structure}

Safe central venous catheterization and management requires standardized education, simulations, and structures for education and instruction [4]. At the Symposium of 127th Japanese Association of Medical Sciences, the science of medical safety focused on abiding by three keywords—-standardization, simulation, and education.

Ultrasound-guided puncture approaches should be aggressively applied to improve the techniques of central venous catheterization. Real-time ultrasound catheterization can visualize both target vein and needle during the procedure. To achieve this skill, simulation training is effective $[5,6]$; however, enough training should be needed to achieve the skill. Institutions and hospitals should introduce the use of mannequins and other educational equipment for puncture simulations. If it is difficult for an institution to obtain such equipment on its own, holding joint training sessions at academic conferences or other venues is recommended. These sessions can also provide opportunities to learn methods for discovering complications quickly. Simulation training should also cover infection control [7].

Simulation training using cadavers in the risky procedures has been recently possible in Japan, and it has been progressing and somewhat shown effectiveness [8]. We recommend educating novice operators using cadaver training at starting clinical training period by experts and educational specialists.

The purpose of central venous catheterization is to improve the therapeutic results of anesthetic management and other procedures while ensuring patient safety. Training programs to achieve this should be designed to improve technical skills and teach safety, infection control, how to obtain informed consent, and explanation skills. Administrators should improve the medical care environment to ensure that this educational structure functions properly.
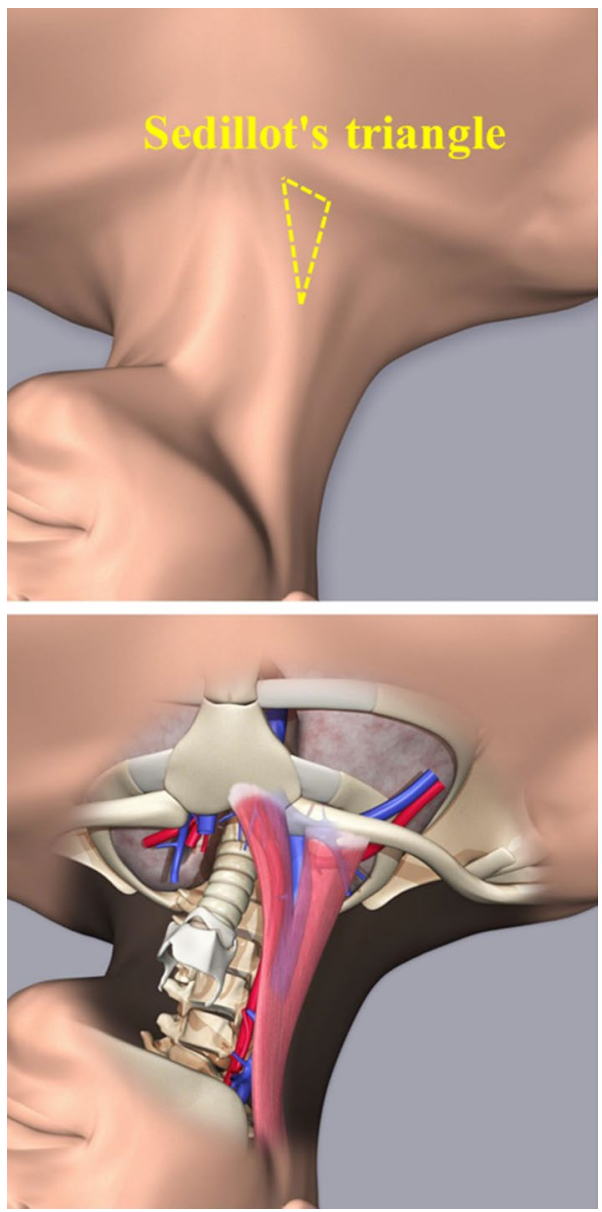

Fig. 1 Sedillot's triangle. Sedillot's triangle comprises the sternocleidomastoid muscle and the clavicle. The triangle can be visually approximated or palpated. In most cases, the internal jugular vein is located within the triangle. Furthermore, insertion and catheterization within the triangle have the benefit of easy handling of the needle and dilator because of the thin tissue layer between the skin surface and the vein

\section{Central venous catheterization}

\section{Understanding the anatomy of the puncture site}

Performing safe catheterization requires not only technical proficiency but also knowledge of the anatomy of the puncture site $[9,10]$. The following summarizes the anatomical characteristics of each puncture site and the areas, where caution is needed.

\section{Internal jugular vein [10]}

The internal jugular vein (IJV) lies at a subcutaneous depth of about $1 \mathrm{~cm}$ within what is known as the Sedillot's triangle on the neck, which is formed by the sternal 
and clavicular heads of the sternocleidomastoid muscle and the clavicle (Fig. 1) [11]. At this location, the IJV runs roughly parallel to the trachea. The central approach, in which a puncture is made from the apex of this triangle toward the ipsilateral nipple, avoids erroneous puncture of the common carotid artery. The operator should remember needle direction not being coincide with the pathway of the IJV. It means that once the needle cannot catch the IJV, never catch the IJV with deeper sticking. When using the central approach and the usual depth of the vein is exceeded, the subclavian artery or the lung can be possibly punctured. Various small arteries lie posterior to the IJV, including the thyrocervical trunk branching from the subclavian artery, the inferior thyroid artery, the transverse cervical artery, and the suprascapular artery as well as the vertebral artery and other vessels, which are all at risk of puncture during this procedure. To avoid mistakenly puncturing one of these arteries, practitioners must avoid puncturing deeply or medially (Fig. 2) [12].

As the IJV approaches the clavicle, it enters the mediastinum; hence, when puncturing in this area, practitioners need to be careful of the medial bend of the IJV. Assuming that the IJV runs in a straight line creates a risk not only of missing the vessel, but also of directing the puncture needle toward the lung.

\section{Subclavian vein [10]}

The subclavian vein (SV) is normally located at the medial third of the dorsal side of the clavicle. To puncture the SV, from this site, aim for a short and narrow gap between the clavicle and the costoclavicular ligament, which is located on the medial side of the first rib. The classic landmark method is direct insertion of the needle sticking from the infraclavicular fossa (an area on the lateral clavicle, where the muscle becomes thin between the lateral margin of the pectoralis major and the medial margin of the deltoid) into the gap between the medial clavicle and medial first rib. Another method aims directly at the medial third of the dorsal side of the clavicle. Whichever is used, puncturing the nearby subclavian artery or the lung, causing pneumothorax, is possible. Damaging both simultaneously results in hemopneumothorax.

Recently, evidence on the usefulness of ultrasoundguided SV puncture (more precisely, infraclavicular axillary vein puncture: IAV puncture) has accumulated [13]. However, at this time, only experienced practitioners have achieved high success rates and low complication incidence rates [14]. Beginners should thus refrain from casually using this puncture method.

\section{Femoral vein [10]}

Femoral vein (FV) puncture is usually described as aiming for an area 2 finger-widths caudal to the inguinal ligament and medial to the femoral artery. Be careful not to pass over the puncture needle cranial to the inguinal ligament. Especially in the right side, if the needle reaches the cranial side of the inguinal ligament, the (external iliac) artery is overlaid on the (external iliac) vein, which makes it easy to mistakenly puncture the artery. If this happens, massive bleeding could occur in the retroperitoneum or abdominal cavity.

\section{Peripherally inserted central catheter}

Traditionally, peripherally inserted central catheter (PICC) is placed in the superior vena cava from a cutaneous vein near the elbow [usually the basilic vein (BV)]. In this area, elbow movements can cause the catheter to rub against the skin, causing phlebitis. Furthermore, catheterization of the small BV near the elbow may inhibit blood flow and cause thrombus formation. Recently, ultrasound-guided puncture
Fig. 2 Anatomical consideration for preventing mechanical complications. a Vessels and nerves surrounding the internal jugular vein. b Magnified figure of the dashed square in part a showing the transparent view of the internal jugular vein and surrounding vessels and nerves. $I J V$ internal jugular vein, $C A$ carotid artery, $S A$ subclavian artery
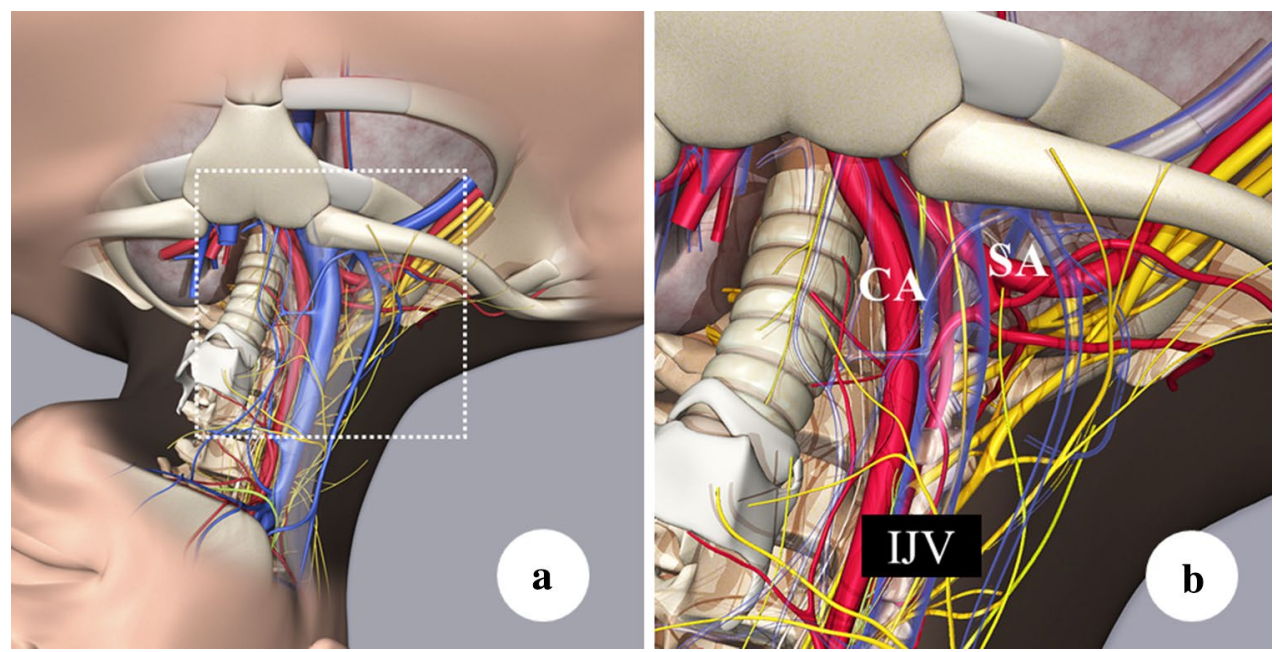
of the BV on the upper arm has become the mainstream form of PICC [15]. The BV on the upper arm is relatively large; thus, blood flow is less likely to be inhibited. However, it is usually difficult to confirm the vein with the naked eye in this area; thus, ultrasound guidance is needed.

\section{Catheterization methods}

\section{Puncture techniques}

Central venous catheterization methods are mainly classified into two [16-18]: the anatomical landmark techniques, which use gross anatomical landmarks on the body surface, and ultrasound-guided techniques, which use ultrasound images.

In ultrasound-guided techniques, a pre-procedure ultrasound examination (pre-scan [18]) is performed before the puncture to observe the area around the target vein in ultrasound images. In the static approach, puncturing is performed after understanding the positional relationships of anatomical landmarks on the body surface. (In Japan, this is called the "map-making method", because it involves marking the skin.) In the real-time approach, puncturing is guided by ultrasound. A pre-scan is always performed even with the real-time approach (if a risk is first revealed in a pre-scan immediately before puncturing, preparations such as re-disinfection would be needed when changing the puncture location).

\section{Pre-procedure ultrasound examination (pre-scan)}

The objective of the pre-scan is not only to confirm the location of arteries and veins (veins are easily flattened by pressure from the ultrasound probe, whereas arteries resist pressure and will cause the probe to throb with the pulse) [18]. Objectives of the pre-scan include (1) assessing whether a vein is suitable for puncture (presence or absence of the target vein, presence or absence of venous thrombosis), (2) assessing risk at the time of puncture (tortuous or deviated veins, positional relationship to arteries, relationship to surrounding tissue), (3) post-procedural risk assessment (vein diameter: catheterization can reduce blood flow in small veins, which can cause thrombus formation or stenosis of the vein) (Fig. 3). A pre-scan is a useful method of risk assessment and should be performed if possible even when landmark techniques are used. When a pre-scan is performed before employing the landmark technique, the position of the target vein is confirmed with ultrasound; thus, this would fall under the ultrasound-guided category (static approach, described below) $[16,19]$. Therefore, this guide recommends ultrasound-guided techniques for all central venous catheterizations.

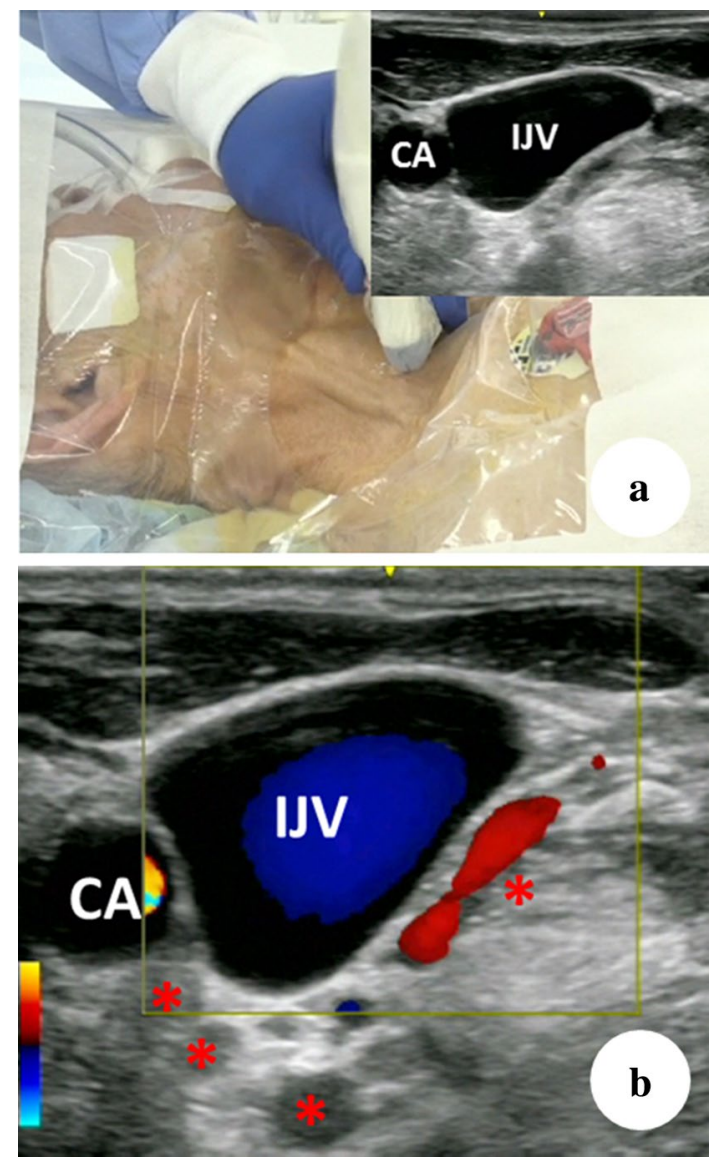

Fig. 3 Small arteries posterior to the IJV. a Ultrasound image of the IJV and CA around the middle of the Sedillot's triangle. b Color Doppler reveals several arteries (asterisk) dorsal to the IJV, which shows the high risk of mistakenly puncturing an artery if the posterior wall of the IJV is penetrated. $I J V$ internal jugular vein, $C A$ common carotid artery

\section{Selection of puncture site [1]}

The target veins for central venous puncture include the IJV, SV (IAV with ultrasound-guided techniques), FV, and upper arm BV (PICC). Table 1 shows the characteristics of each puncture site [17]. The puncture site should be determined by considering the (1) characteristics of the site, (2) risks particular to the patient, and (3) risk assessments based on the pre-scan.

The incidence of catheter-related bloodstream infections is reportedly lowest with the SV and highest with the FV (CDC guidelines) [20], yet other studies have found no difference in infection rates between the sites $[21,22]$. The latest report indicates that catheter-related bloodstream infections occur least frequently with the SV and that there is not much difference between the IJV and the FV [23]. However, many medical institutions are not thorough about infection control; thus, this guide recommends switching from FV catheterization to another central venous route. Furthermore, 
Table 1 Characteristics of each puncture site (adapted from [1]) [1, 15]

\begin{tabular}{|c|c|c|}
\hline Target vein & Advantages & Disadvantages \\
\hline Internal jugular vein & $\begin{array}{l}\text { Vein is easy to identify } \\
\text { Low risk of mechanical complications } \\
\text { Infrequent thrombus formation } \\
\text { Puncturing easy with ultrasound-guided techniques }\end{array}$ & Strong catheter discomfort \\
\hline Subclavian vein & $\begin{array}{l}\text { Little catheter discomfort } \\
\text { Low risk of thrombus formation }\end{array}$ & $\begin{array}{l}\text { Vein is difficult to identify } \\
\text { Easy to mistakenly enter the cranial side on right side } \\
\text { High risk of pneumothorax and/or hemopneumothorax } \\
\text { Risk of pinch-off syndrome }{ }^{\mathrm{a}} \\
\text { Ultrasound-guided puncture techniques are difficult for } \\
\text { inexperienced practitioners }\end{array}$ \\
\hline Femoral vein & $\begin{array}{l}\text { Vein is easy to identify } \\
\text { Puncturing easy with ultrasound-guided techniques }\end{array}$ & $\begin{array}{l}\text { Strong catheter discomfort } \\
\text { High risk of thrombus formation }\end{array}$ \\
\hline PICC & $\begin{array}{l}\text { Lethal complications unlikely } \\
\text { Low risk of infection }\end{array}$ & High risk of thrombus formation \\
\hline
\end{tabular}

${ }^{\text {a }}$ Pinch-off syndrome occurs when the catheter is inserted through the ligament between the first rib and the clavicle, which causes the catheter to be pinched when the arm is moved, leading to poor infusion and eventually even cutting off the catheter. PICC, peripherally inserted central catheter

FV catheterization is not a recommended a site due to the high risk of thrombus formation associated with it.

PICC, which has a low risk of mechanical complications, should be considered for high-risk patients [24].

\section{Risk assessments}

Patient-specific risks include obesity (body mass index $>30$ ) or low body weight (emaciation; body mass index $<20$ ) $[25$, 26], edema [26], a surgical wound [25, 26], pacemaker [26], or infection [26] at the puncture site, radiation therapy [25], coagulopathy (or uncorrected anticoagulant therapy, antiplatelet therapy) [26], and inability to be in the supine position (increased intracranial pressure, heart failure) [26, 27]. The presence of these risks makes central venous puncture even more dangerous; thus, the decision to puncture and the selection of the puncture site should be considered carefully in such cases. The aforementioned PICC is one method of averting risk.

The information needed to assess risk includes the patient's physique (height, weight, outward appearance), general condition (undernutrition, dehydration, presence/ absence of hypovolemia), physiological findings (respiratory sounds), chest radiographs (computed tomography images provide more accurate assessments), complete blood count, blood biochemistry, coagulation function test, medical history, and current disease history (internal medication) (see "Risk factors"). The normal safety criteria for risk of bleeding are platelet count of $50,000 / \mathrm{ml}^{3}$ or higher, prothrombin time international normalized ratio (PT-INR) of 1.8 or less, and activated partial thromboplastin time of no more than 1.3 times the normal upper limit [1]. The decision to puncture should done after fully considering the patient's condition and the risks and benefits. Therefore, a risk assessment must be performed before puncturing.

\section{Procedure of manipulations [28]}

The process is carried out in the following order: (A) monitoring and preparation of for the emergency cart, (B) patient positioning, (C) pre-scan, (D) maximal sterile barrier precautions (MBPs), (E) checking the catheter kit, (F) time-out $[29,30],(\mathrm{G})$ disinfection, $(\mathrm{H})$ puncture and catheterization, and (I) confirmation of the catheter's location.

\section{A. Monitoring and preparation of the emergency cart}

Monitoring is necessary; thus, any mechanical complications that occur due to puncturing (bleeding, pneumothorax, hemopneumothorax, arrhythmia, airway obstruction due to hematoma, cerebral infarction, etc.) can be discovered quickly and treated as soon as possible. It uses peripheral arterial oxygen saturation (pulse oximeter), electrocardiogram, sphygmomanometer, chest stethoscope (follow the JSA Monitor's Guidelines for Safe Anesthesia [31]). When using artificial respiration, capnometry and the graphic monitor of the artificial respirator (airway pressure, flowvolume curve) are useful for detecting unexpected dislocation of an artificial respiration circuit, kinking of the tracheal tube, or other problems that may occur when the patient is covered by sterile drapes. If the patient is conscious, communicate with him/her throughout the procedure. Do not simply try to obtain cooperation from patients, but listen to 
their complaints and statements to help discover complications quickly.

The emergency cart should be stocked the implements needed to secure an airway in an emergency (oxygen mask, tracheal tube, intubation tools, oxygen cylinder, etc.), a defibrillator, critical-care drugs, etc.

\section{B. Patient positioning}

The basic position for puncturing the IJV or SV (IAV) is the Trendelenburg position [32], with the bed angled at about $10^{\circ}-20^{\circ}$. An angle of about $10^{\circ}$ is appropriate when puncturing under general anesthesia [11]. In this position, the IJV is dilated, which makes puncturing easier (the SV is also slightly dilated [33], and air embolism does not occur easily (see "Air embolism")). Adding the Valsalva maneuver increases venous pressure, which is thought to help prevent the needle from penetrating the posterior wall [34]. Setting the ventilator to apply positive end-expiratory pressure at about $5-10 \mathrm{~cm}$ water-column pressure will also help increase venous pressure $[35,36]$. FV punctures are performed in the horizontal or reverse Trendelenburg position [37].

When puncturing the IJV, the neck should be rotated about $30^{\circ}$ away from the puncture site. Over-rotation can create large overlap of the ICV and CA, which increases the risk of mistakenly puncturing the CA [38]. When the sternocleidomastoid is indistinct or if the patient has a short neck, placing a towel or some other object under the shoulder of the side being punctured can help make the bulge of the sternocleidomastoid stand out.

\section{Pre-scan (described above)}

\section{Maximal sterile barrier precautions (MBPs) $[20,28] \mathrm{MBPs}$} are necessary. In MBP, the operator wears a mask, cap (covering all the hair), sterile gloves, and a sterile gown, and the patient is covered with large sterile drapes (see "Infection control in central venous puncture and catheter management").

\section{E. Checking the catheter kit}

Catheter kits are broadly classified into three types depending on the puncture technique used. In the Seldinger technique, a guidewire is inserted after puncturing with a metal needle, then a dilator is used to expand the puncture hole, and finally, the catheter is placed. In the modified Seldinger technique, a needle with a cannula attached is used. After puncturing, the guidewire is inserted through the cannula (i.e., all techniques that use a guidewire can be categorized under the Seldinger technique).

Both techniques are characterized by puncture needles with relatively small diameters. (Usually, needles of $18 \mathrm{G}$ or thinner are used. With ultrasound-guided techniques, even 20 - to 22-G needles can be noticeable, and small needles are favorably used, because they are minimally invasive to the vein.) There is also the through-the-needle/cannula technique (with through-the-cannula technique, a splitting sheath divided into two is used), which uses a large-diameter needle ( $16 \mathrm{G}$ or larger) to puncture, then the catheter is placed directly through the needle or through the cannula (no guidewire is used).

Because mistaken punctures are less invasive with small needles, this guide recommends the Seldinger or modified Seldinger technique.

Ensure that nothing in the catheter kit is defective. Specifically, this includes checking if (1) physiological saline solution can pass through the catheter and (2) the guidewire can be inserted into the puncture needle or dilator. Read the instruction manuals beforehand for any unfamiliar items (this not only saves time during the procedure, but also can help prevent medical accidents from improper usage).

\section{F. Time-out $[29,30]$}

Taking a time-out is important for medical safety (using a checklist is recommended [29]).

The staff should confirm the patient's name, purpose of the puncture, puncture site, patient-specific risks, and disinfection method (presence or absence of allergies), then begin disinfection.

\section{G. Disinfection $[20,28]$}

The puncture site should be disinfected with $1 \%$ chlorhexidine-alcohol or an iodine formulation (povidone-iodine). Before use, the staff should confirm the content of all medical fluids, then dispose of any leftover disinfectants after use (in particular, 1\% chlorhexidine-alcohol is clear; thus, be careful not to mistake it for physiological saline solution). Patients who are allergic to alcohol should be disinfected using an iodine formulation (povidone-iodine). The operator should wash his/her hands for the procedure and carry out MBPs after disinfecting his/her hands with a hand disinfectant (see "Infection control in central venous puncture and catheter management").

\section{H. Puncture and catheterization}

Puncturing is performed as described above, with the ultrasound-guided static approach (the location of the target vein is confirmed in the pre-scan, the positional relationship with anatomical landmarks on the body surface is understood, and the puncture direction and length are estimated) or a real-time approach. With the real-time approach in particular, sufficient training on a simulator should be done before 
performing punctures in the clinical setting (see "Educational structure").

The following summarizes a common method of central venous catheterization, the right IJV puncture.

\section{Static approach (map-making method)}

Confirm the location of the IJV in the Sedillot's triangle during the pre-scan (if necessary, draw a map using a skin pen - this is why the static approach is also called the mapmaking method) (Fig. 4).

1. In the pre-scan, measure the rough distance to the IJV if puncturing at a $30^{\circ}-45^{\circ}$ angle to the skin, and use this as a guide so as to not puncture deeply.

2. Before the main puncture, perform a pilot puncture using a small needle (22-23 G). This is done to ensure that a puncture at the direction and depth confirmed by ultrasound reaches the IJV.

3. Confirm whether the needle has entered the vein by applying slight negative pressure to the syringe to see whether any blood is aspirated into the syringe (backflow of blood).

4. If backflow of blood is observed, remember the puncture direction and length for the main puncture [the red dashed arrow shows the puncture direction (Fig. 4a)].

5. The main puncture uses a larger needle than the pilot puncture, which makes is more difficult to penetrate the anterior wall of the vein and tends to lead to a slightly deeper puncture (Fig. 4b). Puncturing deeper than necessary increases the possibility of complications; thus, sufficient care should be taken. (Be careful not to carelessly puncture deeper than the anticipated puncture length. The absence of backflow of blood even after puncturing to the anticipated depth indicates a strong possibility that both the anterior and posterior walls have been penetrated. If so, slowly withdraw the needle while applying negative pressure. If the posterior wall was penetrated, backflow of blood will arrive once the needle returns to the lumen of the vein.)

6. Examine the force and color of the backflow of blood to check for mistaken arterial puncture. However, if the bevel of the needle is touching the posterior wall of the vein or if a patient has poor circulation (e.g., patient is in state of shock), a decision cannot be made based on the force of the backflow of blood alone [29, 39]. Furthermore, in patients with anemia or those using artificial respiration, the aspirated blood cannot be confirmed to be venous blood based on the color [29, 39].

If mistaken arterial puncture is not accurately assessed and a dilator is inserted when an artery has been punctured, it will increase the damage to the artery and lead to secondary complications. There-

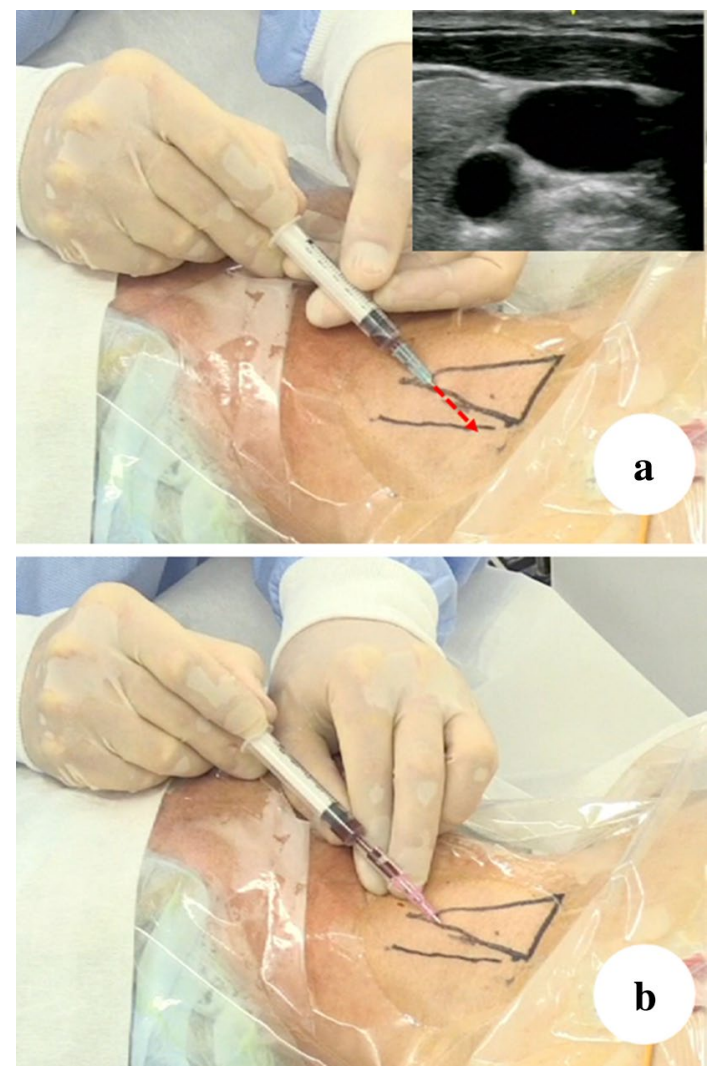

Fig. 4 Static approach. The triangle shows the Sedillot's triangle. The line lateral to the Sedillot's triangle indicates the IJV location confirmed in an ultrasound image. In some cases, the IJV is located outside of the Sedillot's triangle. If the operator inserts the needle immediately above the line, which was the IJV location confirmed in an ultrasound image, the sternocleidomastoid muscle (clavicular head) would likely be penetrated, which may cause difficulty in the insertion of the dilator. When enlarging the route of the guidewire through the thick muscle using the dilator, a patient who is awake may feel discomfort and fear. Furthermore, difficulty in the insertion of the dilator may sometimes cause kinks and aberrant movement of the guidewire from the vein. Even the procedure has been performed safely, the patient will complain of discomfort when they move their neck. If the operator chooses the real-time ultrasound-guided approach in this case, the operator can insert the needle on the line at a relatively cephalad portion, which can prevent the penetration of the sternocleidomastoid muscle, rendering it safer

fore, when using the static approach, use a needle with a cannula (modified Seldinger technique), and once backflow of blood has been confirmed, temporarily insert a guidewire and place the cannula along it. Then, check whether the backflow of blood from the cannula is arterial. If in doubt, take a blood sample for blood gas analysis, attach a small extension tube to measure venous pressure, or attach a pressure monitor to check for an arterial pressure waveform [29] (see "Confirmation procedure to avoid mechanical complications").

7. Once it is confirmed that an artery has not been mistakenly punctured, insert the guidewire. When doing this, 
confirm that there is no resistance to the insertion. Do not insert the guidewire deeper than $20 \mathrm{~cm}$. Inserting the guidewire deeply has the risk of stimulating the heart, which can cause arrhythmia [39, 40]. If arrhythmia occurs, immediately withdraw the guidewire and wait until normal sinus rhythm is restored (sometimes defibrillation may be necessary [41]).

8. When inserting the dilator, hold the posterior end of the guidewire firmly and insert the dilator along the natural angle of the guidewire to prevent the guidewire from bending or being left behind in the body. The dilator should be inserted no more than $5 \mathrm{~cm}$; hence, be careful not to insert it deeper than necessary. (For patients with thick necks, use ultrasound to measure the necessary insertion length, then insert the dilator carefully.) Inserting a dilator deeply could damage the vein $[42,43]$.

9. When inserting the catheter, hold the guidewire firmly to prevent being left in the body. The catheter is placed at a depth of about $13 \mathrm{~cm}$ in a standard adult (12-15 cm, estimated based on height) [44]. There are methods for making accurate estimates based on anatomical landmarks on the body surface and information from chest radiographs [45]. In one simple method, the distance is estimated by adding the length between the needle insertion point (normally, the apex of the Sedillot's triangle on the right neck) and the right clavicular notch (sternoclavicular joint) and where the joint of the manubrium sterni touches the second rib [46].

10. The catheter should be fixed in place correctly according to the instruction manual of the product used.

\section{Real-time approach $[16,17,47]$}

These are broadly classified into two categories based on the positional relationship between the vein and the needle in ultrasound images.

\section{Short-axis out-of-plane technique $[16,17,47]$}

In this technique, the needle is inserted while observing the short axis of the vein; thus, the needle intersects the ultrasound image. It is mainly used for puncturing the IJV and FV. It is advantageous, because positional relationships with arteries are easy to identify, but its disadvantage is that it is difficult to know, where the needle is until it enters the beam plane of the ultrasound scan. In addition, distinguishing between the tip and the shaft of the needle by observing an ultrasound image can be difficult. Therefore, if the needle goes outside the scan plane and goes through the vein, the tip can possibly puncture an artery or the lung.

Figure 5 shows the difficulty in distinguishing between the tip and the shaft of a needle in an ultrasound image. In
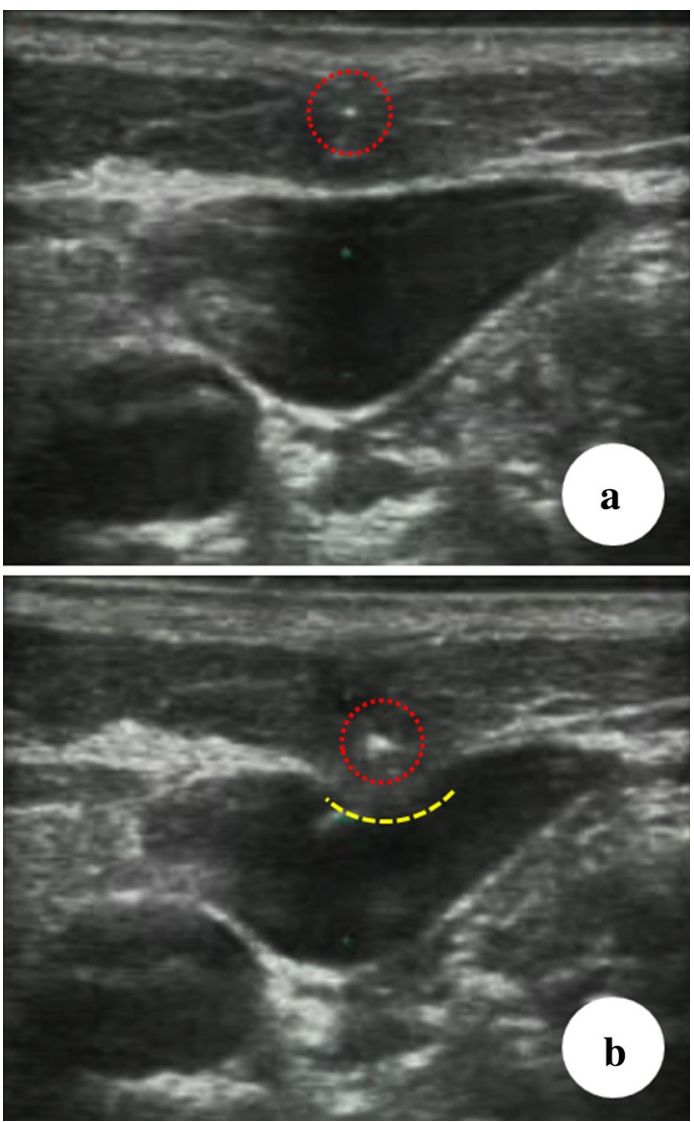

Fig. 5 Pitfall of the short-axis out-of-plane technique. The yellow dotted line shows the dimple-like deformed anterior vein wall. It indicates the needle reaching and pushing the anterior vein wall. The white dot represents the shaft of the needle. a The needle tip (dotted red circle) is on the ultrasound beam. b The ultrasound image depicts part of the needle shaft (dotted red circle)

Fig. 5a, the needle tip (dotted red circle) is on the ultrasound beam. In Fig. 5b, the ultrasound image depicts part of the needle shaft (dotted red circle). The intensity of the shaft is greater than that of the tip in Fig. 5a, which makes it easy to make mistakes. A depression in the anterior wall of the vein (yellow dotted line) can be observed. This shows that the needle is outside the ultrasound beam plane and is touching the anterior wall.

If the puncture is performed only by confirming the vein's short axis, there is a possibility that the puncture direction will differ from the path of the vein. This would cause the needle to penetrate the vein diagonally, which could lead to a mistaken puncture.

To make up for these disadvantages, perform a careful pre-scan to confirm the path of the vein and ensure the puncture follows this path. It is also important to constantly confirm the location of the needle while being inserted. It is important to understand that the needle tip is not recognized from its appearance, but that it is the first point of brightness 
to enter the ultrasound beam plane. As shown in Fig. 5, if a point of brightness is seen throughout the insertion of the needle, it is highly likely this is not the tip of the needle but the shaft.

\section{Short-axis out-of-plane theory $[11,12,47]$}

Two types of scans need to be combined to grasp the path of the target vein.

First, a sweep scan is performed, in which the probe sweeps over the path of the vessel like a broom (about $2 \mathrm{~cm}$ ). The probe should be manipulated so that the vein is always at the center of the ultrasound image (Fig. 6).

The next technique involves swinging the probe like a fan (about $30^{\circ}$, Fig. 7). Similarly, the probe should be manipulated so the vein is constantly at the middle of the ultrasound image (Fig. 7).

Combining these two scans ensures that the probe is completely perpendicular to the vein. Therefore, if the puncture is directed toward the center of the probe, the needle will advance toward the center of the vein (Fig. 8).

It is important to confirm the location of the needle tip to ensure that the posterior wall is not penetrated (described above). In the short-axis out-of-plane technique, the vein intersects the scan plane; thus, the needle is guided into the ultrasound image. The needle tip is being confirmed intermittently in this method; thus, it is not strictly a real-time puncture. The position of the needle tip is not confirmed by depicting it in real time, but is based on theory (Fig. 9).

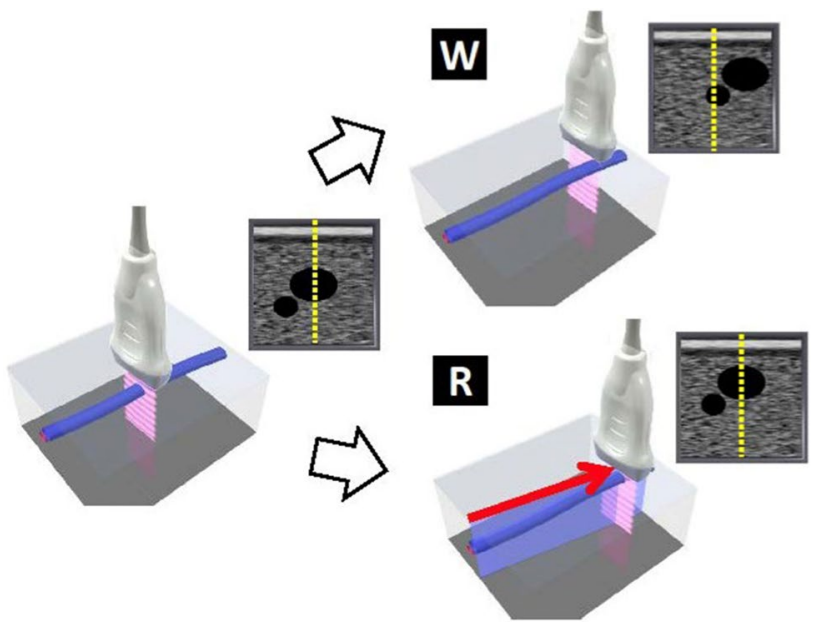

Fig. 6 Sweep scan technique (reproduced from [12]). W, the scan is not following the path of the vessel; thus, the target vein, which was at the center of the image, moves toward the edge of the image; $\mathrm{R}$, when the scan follows the path of the vessel, the vein stays at the center of the image. Conversely, if the vein remains at the center of the image during the sweep scan, the scan is following the path of the vessel

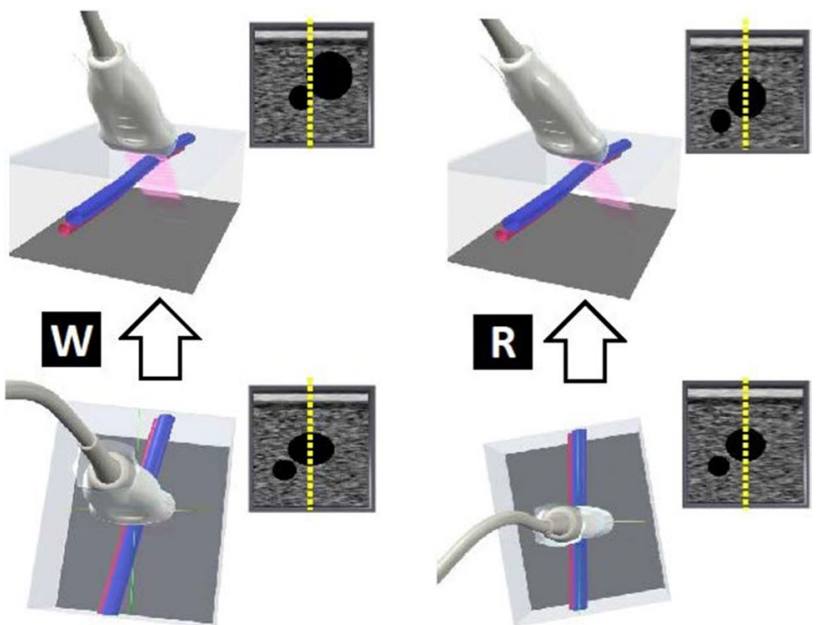

Fig. 7 Swing scan technique (reproduced from [12]). W, if the probe is not perpendicular to the long axis of the vein, the vein will move to the edge of the image when the probe is swung; $R$, if the probe is perpendicular to the long axis of the vein, the vein will stay at the center of the image when the probe is swung. Conversely, if the vein stays at the center of the image during a swing scan, the probe is perpendicular to the path of the vessel

\section{Long-axis in-plane technique $[12,48]$}

In this technique, the needle is advanced inside the ultrasound scan plane while observing the long axis of the vein. Unlike the short-axis out-of-plane, puncturing is truly performed in real time. Although this technique can be applied clinically with the IJV if a small probe is used, it can be difficult to use regular-sized probes on the necks of small adults due to lack of space to manipulate. This technique is particularly useful with the IAV. With the long-axis in-plane technique, penetration of both the anterior and posterior walls of the vein does not occur very often, but the needle may penetrate the vein if the needle is directed from the anterior wall toward one of the lateral walls [48]. Depicting the long

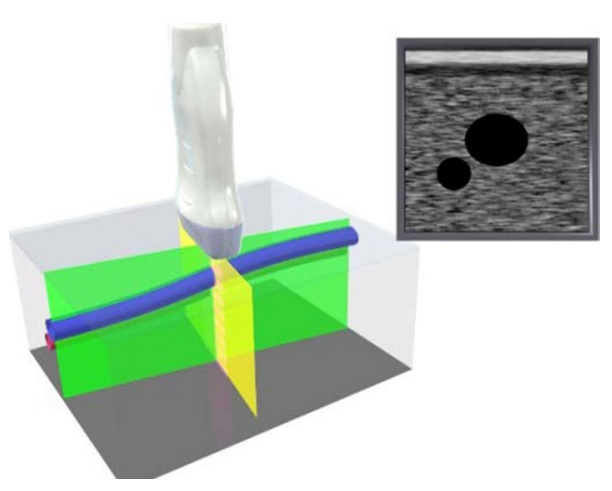

Fig. 8 Probe placed perpendicular to the path of a vessel (reproduced from [12]) 


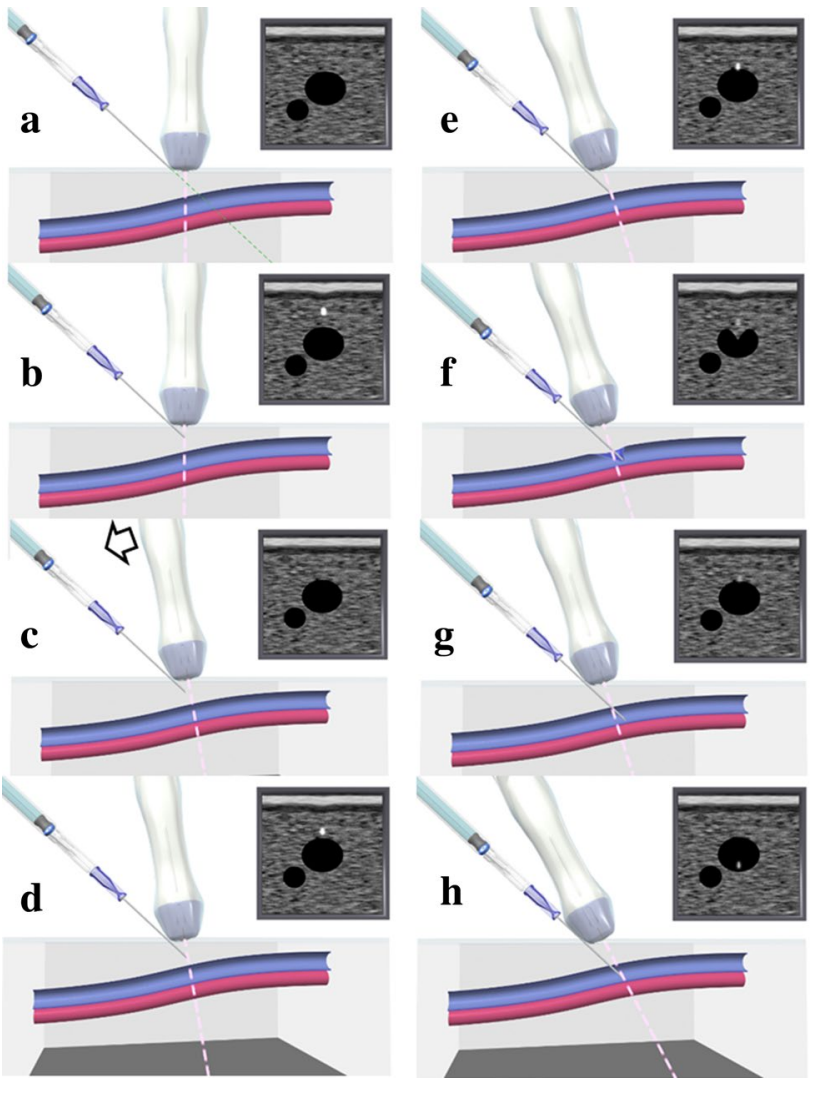

Fig. 9 Manipulation of the needle and confirmation of the tip (reproduced from [12]). a When the needle is guided into the scan plane, b the first point of brightness to appear is the needle tip. c Next, the scan plane is moved slightly forward, which causes the point of brightness to disappear. d The needle is again advanced slowly into the scan plane, which causes a point of brightness to reappear. e Repeating this manipulation, the needle gradually reaches the target vein. $\mathbf{f}$ When the needle tip touches the anterior wall of the target vein, it creates a small dimple, turning the vein into a heart shape. Pushing the needle into the vein with a snapping motion allows it to move a short distance so that it only penetrates the anterior wall. (Venous pressure is normally low; thus, if the needle is pressed slowly into the anterior wall and the tip reaches the posterior wall, it could penetrate the posterior wall as well.) g Finally, the anterior wall returns to its original shape, the shaft comes into view, and the tip can no longer be seen. $\mathbf{h}$ To see the tip, move the scan line forward

axis of the center of the vein can prevent a needle directed toward a lateral wall from penetrating the vein [48] (Fig. 10).

\section{Confirmation of guidewire position $[16,29]$}

In the real-time approach, ultrasound is used not only during puncturing, but also to confirm that the guidewire has been placed correctly inside the vein. If it is difficult to depict a small wire using ultrasound, shaking the guidewire: repeatedly inserting and withdrawing the guidewire in small amounts (about $5 \mathrm{~mm}$ ) makes confirming the location easy [47]. If the wire gradually comes into contact with the

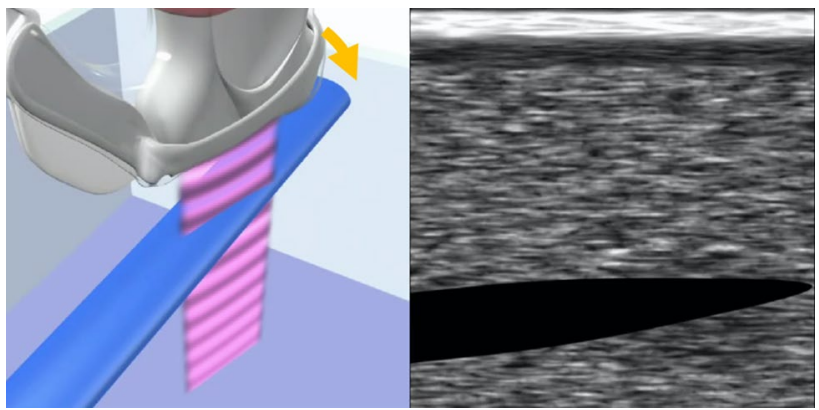

Fig. 10 Long-axis in-plane technique. To perform the procedure safely, the operator should identify the center of the vein using the scan called the "side-scape scan technique" [48], which includes the following steps: First, turn the distal part of the probe to the right until the right lateral wall is identified (the yellow arrow shows the turning direction). The lateral wall appears to have a bullet shape because of the merging of the anterior and posterior walls. Second, turn the distal part of the probe to the left, and the left lateral wall can then be identified. The operator can estimate the distal center of the vein and then repeat the same motion at the proximal side of the vein to estimate the proximal center of the vein. Third, the operator can obtain the longitudinal ultrasound view of the center of the vein. Finally, with the needle guide and/or personal skills, advance the needle within the ultrasound beam, which can prevent "anterior to lateral wall penetration."

posterior wall of the vein, it will not penetrate the posterior wall (Fig. 11; see "Main mechanical complications and their countermeasures").

If a guidewire is mistakenly inserted into a vessel it is not intended for, the vein could be damaged when the dilator is inserted. For example, a guidewire inserted from the right IJV may mistakenly migrate into vessels such as the right SV, left brachiocephalic vein, or azygos vein. In such situations, ultrasound can be used to confirm the position of the guidewire and follow it centrally as much as possible. However, while an unintended insertion into the right SV can be confirmed, it can be difficult to detect mistaken insertion into the left brachiocephalic vein or azygos vein using ultrasound. Therefore, the dilator should be inserted gently,

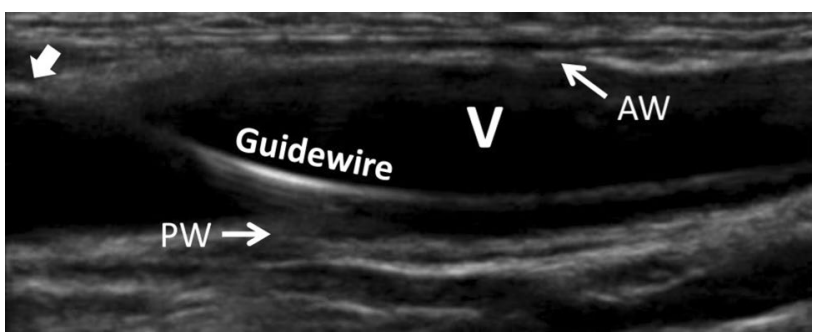

Fig. 11 Confirmation of the guidewire. The white arrow indicates entering point of the guidewire into the vein. $V$ long axis of the internal jugular vein, $A W$ anterior wall of the internal jugular vein, $P W$ posterior wall of the internal jugular vein. The white arrow indicates entering point of the guidewire into the vein 
and the manipulations should be halted if any abnormal resistance is felt. After catheterization, carefully confirm, via a chest radiograph, for example, that the catheter was not inserted into an unintended vessel.

\section{Methods of confirming catheterization [49]}

Confirm that the catheter is placed correctly in the vein and that the tip is in the desired position using a chest radiograph. Ideally, the tip of the catheter should be roughly parallel with the wall of the superior vena cava, caudal to the inferior margin of the clavicle, between the third rib and the fourth/fifth thoracic vertebra, and cranial to the bifurcation of the trachea or right primary bronchus (Fig. 12). The bifurcation of the trachea is usually positioned cranial to the pericardial reflection; thus, it is best if the tip of the catheter is always cranial to this. If perforation due to erosion of the vessel wall occurs, a mediastinal hematoma may occur, transfusion fluid may enter the chest cavity cranial to the pericardial reflection, or cardiac tamponade may occur caudal to this area (see "Main mechanical complications and their countermeasures").

Catheters inserted from the left IJV need to be parallel to the vessel wall. If the tip of a catheter inserted from the right IJV is in zone A, it should be pulled back to zone $\mathrm{B}$. In this case, zone B is the optimal location for the catheter tip.

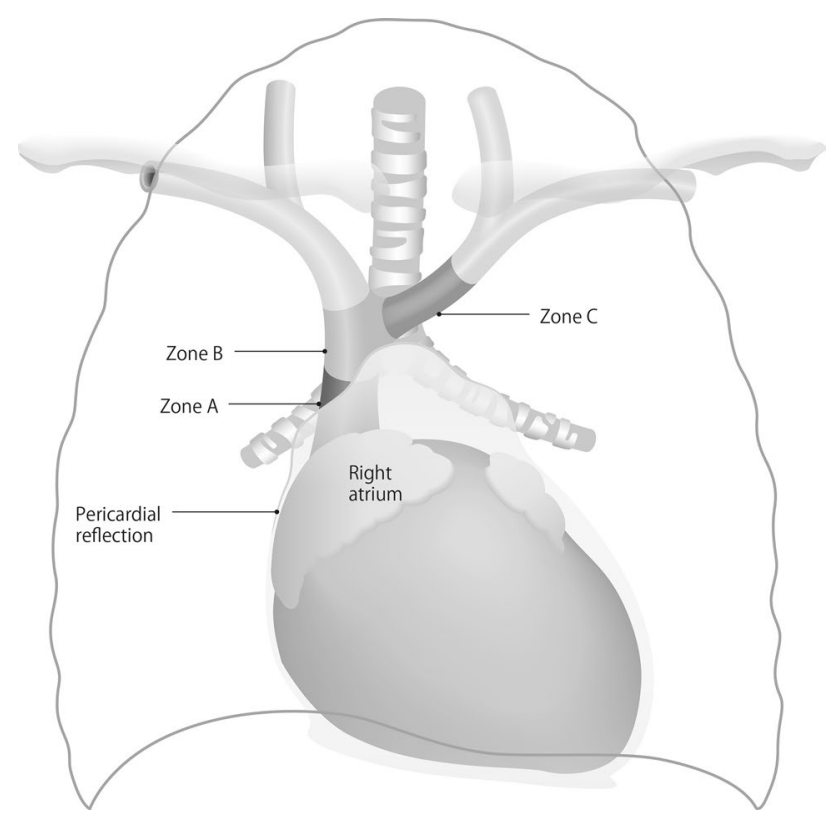

Fig. 12 Optimal catheter placement (modified from [49]). Zone A, inferior portion of the superior vena cava up to the superior portion of the right atrium; zone B, portion of the right innominate vein confluent with the left innominate vein and the superior portion of the superior vena cava; zone $C$, innominate vein peripheral to the superior vena cava

\section{Confirmation procedure to avoid mechanical complications}

The spread of ultrasound devices has reduced the frequency of mechanical complications. However, critical situations can arise when mechanical complications occur. In a 2004 report, the central venous catheter-related mechanical complications with the highest mortality rates were pulmonary artery damage, hemothorax, cardiac tamponade, and air embolism, in that order [50]. Any of these complications could lead to death or a critical situation. Great care should thus be taken to ensure these complications in particular do not occur. To achieve this, place the catheters in the appropriate location and ensure that a dilator or catheter is not mistakenly inserted into an artery. Ultrasound-guided central venous catheterization has been reported to reduce the frequency of mistaken arterial puncture [1, 29].

The preferred confirmation methods for ultrasoundguided central venous catheterization are shown in the following figures. Figure 13 addresses the static approach. Basically, ultrasound images are not used during puncture in the static approach; thus, it is important to check that the puncture needle is definitely in the vein. Inserting a cannula into the vessel allows manipulations to be performed stably.

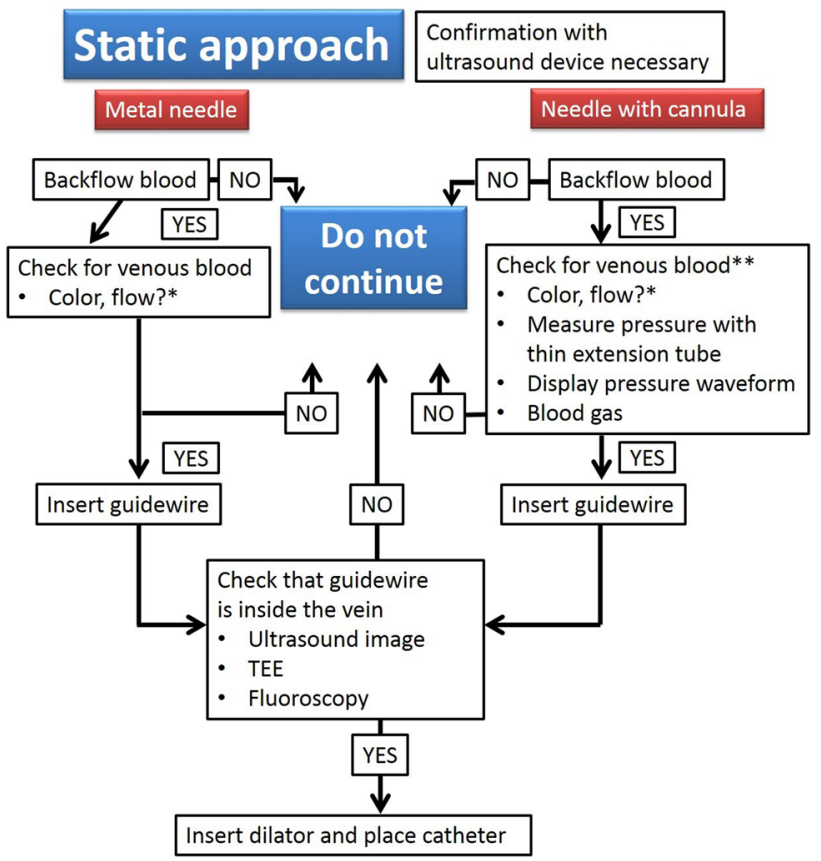

Fig. 13 Central venous catheterization methods with a static approach. *Venous blood cannot be confirmed using only the color or flow of backflow blood. **Insert the guidewire about $10 \mathrm{~cm}$ and insert the cannula up to its base, then confirm by attaching an extension tube to measure pressure, display a pressure waveform, or perform blood gas analysis. TEE, transesophageal echocardiography 
It is possible to mistake arteries for veins with signs such as the color and force of backflow blood with hypoxemia, extremely high oxygen partial pressure, or hypotension, such as in states of shock. It is easy to measure pressure by attaching a small extension tube, by displaying the pressure waveform to confirm the presence of a venous pressure waveform, or by taking a blood sample for blood gas analysis to confirm venous blood. One of these methods is necessary to confirm that the needle is inside a vein. As described in the section on puncture techniques, as it can be difficult to confirm that the punctured vessel is a vein with the metal needles that are used in the static approach, the use of a cannula needle is recommended. Therefore, if a metal needle is selected with the static approach, ultrasound images or other examinations should be used to confirm that the inserted guidewire does not penetrate the posterior wall and is traveling inside the vein. Even if a cannula needle is used, it is preferable to confirm through ultrasound images or other examinations that the inserted guidewire does not penetrate the posterior wall and is traveling inside the vein.

Figure 14 addresses the real-time approach. With this approach, ultrasound is used to confirm that the needle is inside the vein, regardless of whether a metal or cannula needle is used. With a metal needle, once the color or force of the backflow blood indicates the presence of venous

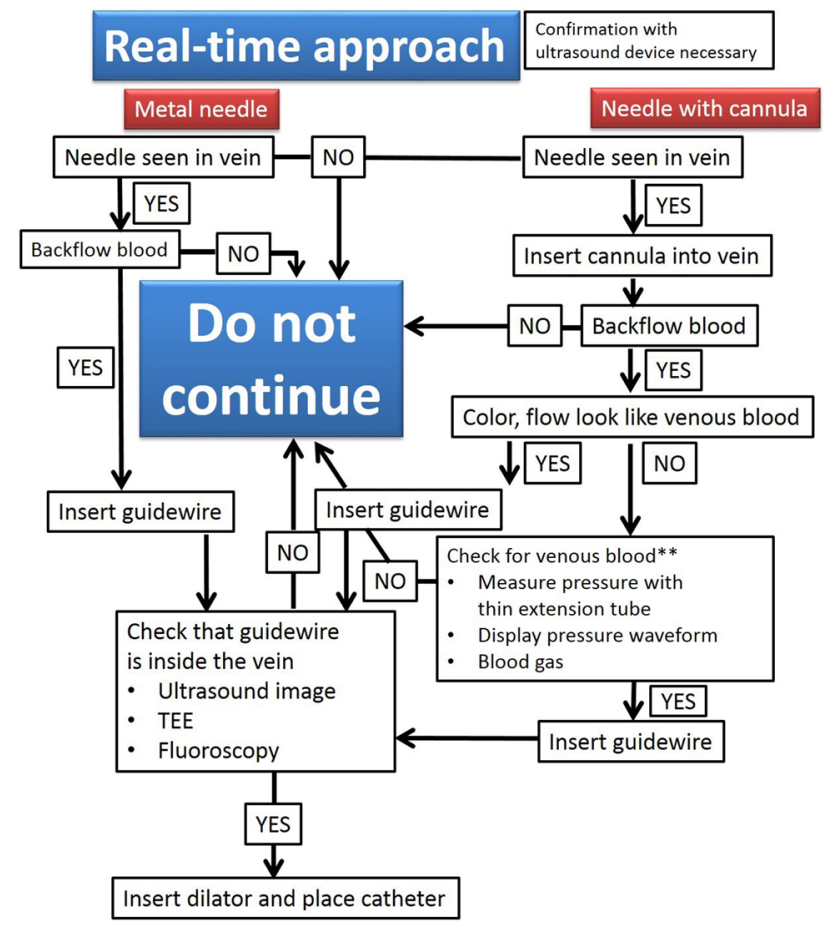

Fig. 14 Confirmation of central venous catheterization with real-time approach. **Insert the guidewire about $10 \mathrm{~cm}$ and insert the cannula up to its base, then confirm by attaching an extension tube to measure pressure, display a pressure waveform, or perform blood gas analysis. TEE, transesophageal echocardiography blood, the guidewire can be inserted. After confirming in the ultrasound images that the guidewire did not penetrate both the anterior and posterior walls of the vein and is traveling inside the vein, the dilator can be inserted. If a cannula needle is used, once the color and force of the backflow blood indicate it is venous blood, the guidewire can be inserted. Then, similar with a metal needle, after confirming in ultrasound images that the guidewire did not penetrate both the anterior and posterior walls of the vein and is traveling inside the vein, the dilator can be inserted. If it is difficult to make a decision based on the backflow blood, insert the guidewire about $10 \mathrm{~cm}$ and insert the cannula up to its base, then, as in the static approach, confirm the presence of venous blood by attaching an extension tube to display a pressure waveform or for blood gas analysis. Then, insert the guidewire. After this, confirm in the ultrasound images that the guidewire does not penetrate both the anterior and posterior walls of the vein and is traveling inside the vein, then insert the dilator. Figures 13 and 14 show ultimately only safety examples; thus, each institution should reference these to establish methods for confirming that a dilator has not been mistakenly inserted into an artery.

Figure 15 shows the extreme importance of confirming in ultrasound images or other methods that the guidewire does not penetrate both the anterior and posterior walls of the vein and is traveling inside the vein. The following describes the methods for confirming in ultrasound images that the guidewire is traveling inside the vein. After inserting the guidewire, the short axis will look like the image in Fig. 15a. However, it cannot be declared that the guidewire is traveling inside the vein from this alone. This is because the possibility that the guidewire has penetrated the vein cannot be ruled out. Thus, short-axis images must be observed by moving the ultrasound probe slowly from the guidewire puncture site toward the heart to around the clavicle. This will show that, in most cases, if the guidewire penetrates the skin and enters the vein, it proceeds almost as if it is in contact with the vessel wall. Next, if possible, the tip of the guidewire should be depicted in a long-axis image. As shown in Fig. 15b, if it can be confirmed that the guidewire tip is traveling along the vein parallel to it and without any changes in angle, it is acceptable to believe that it has not penetrated the vein. This confirmation procedure is extremely important for preventing mechanical complications.

\section{Main mechanical complications and their countermeasures}

\section{Arterial puncture and hematoma}

Central venous catheterization rarely accompanies the formation of hematoma. However, with the jugular vein, and 
Fig. 15 Confirmation of guidewire location using ultrasound images. The patient was a 6-month-old infant. The guidewire can be seen inside the internal jugular vein in the short-axis image (a). However, this alone cannot determine if the guidewire tip is inside the vein. The long-axis image shows that the guidewire path gradually becomes parallel to the vessel wall (b)

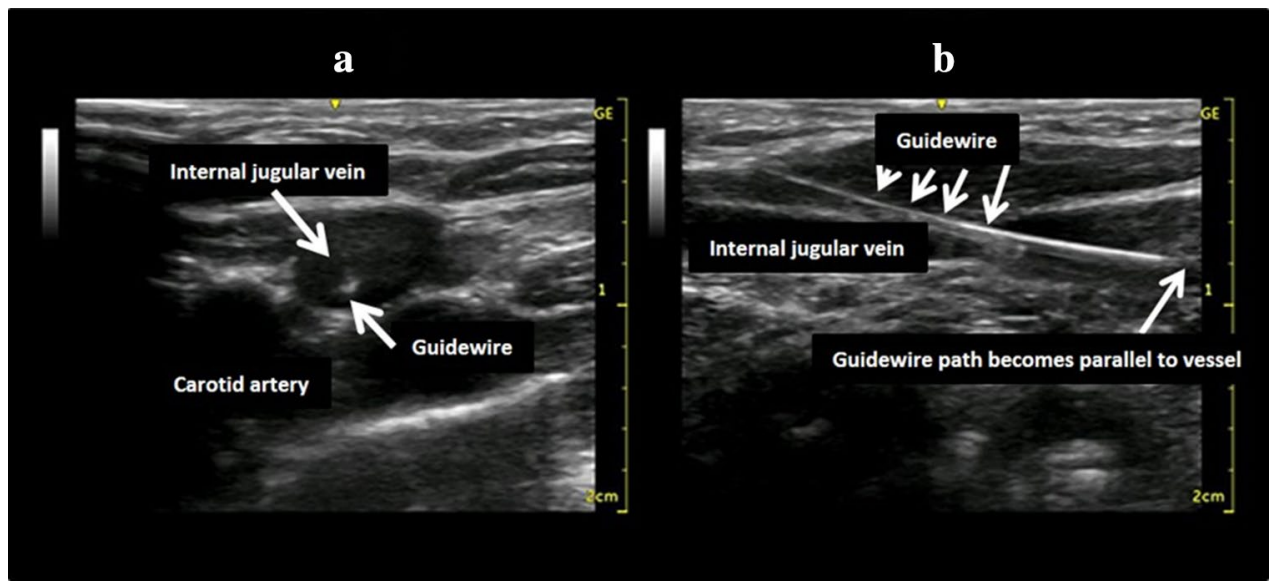

particularly when the carotid artery is mistakenly punctured, the formation of a hematoma may block the upper airway. If an artery is mistakenly punctured during SV puncture, external compression to stop the bleeding can be difficult to apply. If a catheter of $7 \mathrm{Fr}$ (equivalent to a diameter of $2.3 \mathrm{~mm}$ ) or less is inserted into an area, where compression is possible and the catheter can be withdrawn and external compression applied for $10 \mathrm{~min}$, then it can be withdrawn with no problem. In other words, if an artery is mistakenly punctured by a needle thinner than $14 \mathrm{G}$ (equivalent to a diameter of $2.1 \mathrm{~mm}$ ), hemostasis may be possible via compression. However, if a catheter or dilator larger than $7 \mathrm{Fr}$ is inserted into an artery or a vessel for which compression is not possible, a cardiovascular surgeon should be brought into withdraw the catheter safely $[1,51]$; otherwise, cerebral infarction, arteriovenous fistula, or hemothorax might result.

\section{Pneumothorax}

Understand the local anatomy and do not insert puncture needles into risky areas. When performing catheterization, diagnose any cough, chest pain, or respiratory difficulty from auscultatory findings and chest radiographs. Immediately after catheterization, a normal chest radiograph will not rule out pneumothorax. Delayed pneumothorax sometimes occurs. In most cases, pneumothorax that fills about $30 \%$ or less of the chest cavity does not present with clinical symptoms and does not normally require drainage [1]. Recent findings have indicated that ultrasound devices can be useful in diagnosing pneumothorax [52].

\section{Hemothorax, mediastinal hematoma, pleural effusion, and cardiac tamponade}

Particular caution is warranted in patients who require multiple punctures. Subcutaneous diffusion of transfusion fluid can result from catheters during insertion or clinical use or from damaged blood vessels, which can create local tissue edema. Extravasation of fluid that has been injected into a vein may cause pleural effusion. Moreover, punctures in the pericardium can cause cardiac tamponade, which has a high mortality rate. These require drainage of the chest cavity, mediastinum, or pericardium.

\section{Air embolism}

This is caused when air mistakenly enters the vessel through a puncture needle or open catheter end exposed to the open air. Puncturing should be performed in a head-down tilt or the Valsalva maneuver should be added, if necessary, in horizontal supine position. After a catheter is removed, air can be drawn into the puncture hole to cause an air embolism. Therefore, after removing the catheter, cover the puncture site with a transparent dressing or other covering [53, 54].

\section{Arrhythmia}

Mechanical stimulation from a guidewire or catheter can cause arrhythmia, including supraventricular arrhythmia and ventricular fibrillation. Rarely, a patient can transition to continuous ventricular fibrillation when a catheter is removed. In such an event, immediate defibrillation should be performed.

\section{Local nerve damage}

Local nerve damage related to catheterization can present as mechanical injury, nerve compression from a hematoma, or neurotoxicity from medical fluids leaking outside a vessel.

\section{Rare complications}

Rare complications include brachial plexus injury, thoracic duct damage, or chylothorax from left IJV or left SV 
puncture. Knotting of the catheter, a guidewire being left behind, accidental removal, femoral nerve damage from $\mathrm{FV}$ puncture, abdominal cavity puncture, and retroperitoneal hematoma may also occur.

\section{Frequency of complications}

Table 2 shows the frequencies of complications by puncture site with the classic landmark approach without using ultrasound images and with the ultrasound-guided real-time approach [13, 55-57].

The results of a meta-analysis showed that catheterization failures were significantly less frequent for the IVJ and SV with the ultrasound-guided approach than with the landmark approach, but the difference for the FV was not significant [58]. PICC can also be useful in patients such as those with the risk factors discussed below.

Catheter insertions and removals should be recorded on the hospital's designated forms and surveys should be performed to ensure that central venous catheterization is monitored for safety.

\section{Risk factors}

Patient risk factors for mechanical complications include underlying disease, comorbidities, tendency to bleed due to regular medication or other factors, high risk for thromboembolism due to arteriosclerosis, and changes to the anatomical pathways of vessels due to surgery or bone fracture.

Factors that can increase moderate levels of risk include (1) puncturing the site of a previous central venous catheterization, (2) history of local radiation therapy, (3) history of median sternotomy, (4) recent myocardial infarction, (5) thrombocytopenia, (6) venous thrombosis at the puncture site, (7) fibrinolytic therapy, and (8) an anxious patient. Factors that can increase mild levels of risk include (1) abnormal weight/height ratio, (2) severe obesity, (3) prolonged coagulation time, (4) artificial respiration with high airway pressure, (5) moderate to severe arteriosclerosis, (6) sepsis, (7) ventricular arrhythmia, (8) pulmonary emphysema or chronic obstructive pulmonary disease, and (9) hypovolemia [59].

\section{Infection control in central venous puncture and catheter management}

\section{Types of catheter-related infections}

The US Centers for Disease Control and Prevention categorizes catheter-related infections as follows [60]:

1. Catheter colonization: There are no clinical symptoms, but a culture from the catheter surface shows more than 15 colony-forming units (CFUs) in a semiquantitative culture or more than $100 \mathrm{CFUs}$ in a quantitative culture.

2. Local catheter-related infection:

- Exit-site infection: There are no symptoms of a bloodstream infection, but inflammatory symptoms such as erythema, swelling, pain, or purulent exudate are observed within $2 \mathrm{~cm}$ of the puncture site.

- Tunnel infection: There are no symptoms of a bloodstream infection, but signs of an infection are observed in the subcutaneous tissue along the catheter insertion route at least $2 \mathrm{~cm}$ from the puncture site.

- Pocket infection: This occurs in the subcutaneous pocket of an implanted port system. There are no signs of a bloodstream infection, but there are subcutaneous signs of infection or inflammation, where the port is embedded.

3. Infusate-related bloodstream infection: This is a bloodstream infection in which no other infections are present, but the same bacterium is detected from the injection instruments and materials and from a blood culture.

4. Catheter-related bloodstream infection: This is a bloodstream infection in which the same bacterium is detected from the catheter and a blood culture. If the infection is detected less than $48 \mathrm{~h}$ after catheterization, it is classified as a catheter-associated bloodstream infection (little relation to the catheter).
Table 2 Complications by puncture site with the classic landmark approach and ultrasound-guided real-time approach [13, 55-57]

\begin{tabular}{lllll}
\hline Site & Method & $\begin{array}{l}\text { Arterial punc- } \\
\text { ture (\%) }\end{array}$ & Hematoma (\%) & Pneumothorax (\%) \\
\hline \multirow{2}{*}{ All } & Landmark & 6.9 & 8.2 & 3.1 \\
& Ultrasound-guided & 1.4 & 1.6 & 1.3 \\
\multirow{2}{*}{ Internal jugular vein } & Landmark & $5.8-10.6$ & $8.4-9.1$ & $2.4-3.0$ \\
& Ultrasound-guided & $0.3-1.1$ & $0.2-1.2$ & $0-1.2$ \\
Subclavian vein & Landmark & 6.2 & 4.6 & 3.7 \\
& Ultrasound-guided & 2.0 & 1.5 & 0.7 \\
\hline
\end{tabular}




\section{Recommendations for the central venous catheterization environment}

1. Perform central venous catheterization in a place, where sterile manipulations can be performed.

2. Use a standardized catheter set.

3. Have an assistant during central venous catheterization.

4. Use a checklist or protocol for placing and maintaining catheters.

5. Conduct simulation training for central venous catheter insertion.

At present, there is not sufficient evidence for items 1-3 $[2,9]$. Items 4 and 5 have been shown to reduce catheterrelated infections and complications [61-64].

\section{Recommendations for preventing infections}

1. Intravenous antibiotic prophylaxis should not be done routinely. However, this may be administered on a caseby-case basis for immunocompromised patients or highrisk neonates $[65,66]$.

2. Maximal sterile barrier precautions (MBP): this includes hand washing, wearing of mask, cap, sterile gloves, and a sterile gown, and covering of the patient with large sterile drapes. Although the results of randomized trials on MBP have been unclear [67], observational studies have indicated that they reduce catheter-related bloodstream infections [62, 63, 68, 69].

3. Skin disinfectants: use $1 \%$ chlorhexidine-alcohol or $10 \%$ povidone-iodine [70]. Side effects such as contact dermatitis, sensitivity reactions, and anaphylaxis have been reported with chlorhexidine; thus, alcohol solutions or aqueous solutions are currently used. Results regarding the skin toxicity of chlorhexidine in neonates of less than 44 week gestation are unclear; thus, each institution must make its own clinical decisions [29].

4. Antimicrobial-impregnated central venous catheters: antimicrobial-impregnated central venous catheters should be used appropriately in consideration of the latent risk of the expression of resistant bacteria and the expensive cost. They should not be used in patients allergic to antibiotics, because there are reports of anaphylactic shock due to antimicrobial-impregnated central venous catheters [71, 72]. The use of chlorhexidine/ silver sulfadiazine —or rifampicin miconazole-impregnated central venous catheters reduces the risk of catheter-related bloodstream infections [73]. In Japan, only minocycline-rifampicin-impregnated central venous catheters are available. This type of catheter can prevent catheter-related bloodstream infections in the intensive care unit better than chlorhexidine/silver-sulfadiazineimpregnated central venous catheters [74].
5. Closed infusion system: the use of a closed infusion system can reduce the mortality rate of sepsis caused by catheter-related bloodstream infections [75]. In contrast, the incidence of catheter-related bloodstream infections rises with open three-way stopcocks [76]. If an open three-way stopcock is used, wipe the ports thoroughly with cotton soaked in a disinfectant (chlorhexidine, povidone-iodine, $70 \%$ alcohol) for at least $15 \mathrm{~s}$ [77, 78]

6. Puncture site selection: the puncture site should be determined based on clinical necessity. Contaminated areas (infected skin, burns) and areas that may be contaminated (groin, near the tracheostomy, surgical wounds) should be avoided. There is no significant difference in catheter colonization rates and catheter-related bloodstream infection incidence between the IJV and the SV [79-81]. The catheter colonization rate of the FV was higher than that of the SV catheterization, although the catheter-related infection rate was not different between the two veins [82].

7. Covering the puncture site: bio-occlusive dressings are preferred for covering puncture sites to prevent infections. Chlorhexidine-impregnated sponges in catheter dressings can prevent catheter-related infections, and changing the frequency of changing dressings from every 3 days to every 7 days is recommended [83].

8. Duration of catheterization: the duration of central venous catheterization should be based on clinical necessity. Longer periods of catheterization increase the risk of infection, but there is no standard for duration. The need for keeping the catheter should be evaluated daily, and catheters that are no longer needed should be removed immediately. Leaving in place a catheter that is no longer needed may cause catheter-related bloodstream infections. Even regularly replacing a central venous catheter does not reduce the frequency of catheter-related bloodstream infections [84]. The infection rate does not differ between using a guidewire to replace a central venous catheter and replacing it by making a new puncture. Replacing catheters using a guidewire every 3 days and every 7 days were compared, but no significant difference was observed in the formation of colonies on the catheter tip $[85,86]$. The catheter puncture site should be checked daily for signs of infection, and if any are found, the catheter should be removed and the catheterization site changed. If a catheter-related infection is suspected, it is better to change the puncture site than replace the catheter with a guidewire [29].

9. Drug administration and blood aspiration from a central venous catheter: when administering medication or aspirating blood from a central venous catheter, threeway stopcock ports should be wiped thoroughly with an appropriate disinfectant before use [29]. After use, threeway stopcocks should be capped. Access ports may also 
be used. Using needleless connectors can reduce the incidence of port infections [87].

\section{Cautionary notes specific to pediatric central venous catheterization}

\section{Caution during puncture}

- Ultrasound-guided techniques are recommended for children with small veins that are difficult to puncture. Select a thinner, lighter probe than for adults. A linear probe or hockey-stick probe can be used.

\section{Recommended catheters}

- Catheter kits 3-4 Fr and $5 \mathrm{~cm}$ long are available for newborns. Select a size that is suitable for the patient's physique.

\section{Cautionary notes for preparation}

- The position of the patient should be stable. In smaller children, it is especially important to secure an area for puncture manipulations. For instance, placing a pillow under the neck or using tape to extend the neck can help secure an area to operate.

- The lumen of small vessels easily collapses under the weight of an ultrasound probe or a needle. Creating traction on the skin by taping around the puncture site, such as on the mandible or above the clavicle, can help maintain skin tension and reduce pressure on the vessel during the procedure [88].

\section{Anatomy}

- The IJV is often located lateral and anterior to the CA, but may also be directly superior (3.2\%), laterally parallel (3.2\%), or lateral and posterior (1.4\%) to it [89].

- The vertebral artery runs medial and posterior to the IJV; hence, there is a risk of arterial puncture. Compared to adults, children's vertebral arteries are larger compared with their IJV and the distance from the skin to the vertebral artery and from the vertebral artery to the IJV is shorter [90].

\section{Practical puncture techniques}

- In low-birth weight infants, use a syringe smaller than that used in adults to confirm backflow of blood.

- The pressure on the vessel from the puncture needle can cause the anterior wall of the vessel to stick to the pos- terior vessel or collapse the lumen, which can lead to both the anterior and posterior walls being penetrated simultaneously, and backflow of blood may not appear despite vein puncture. Pulling the needle back will create a space between the anterior and posterior walls and cause the vessel lumen to expand and fill with blood; thus, if the vessel has been penetrated, backflow of blood may appear in the process of withdrawing it.

- It can be difficult to differentiate arterial and venous blood by color in patients with cyanotic heart disease. Confirm venous residence of the wire using ultrasound imaging, or if this cannot be determined via ultrasound, measure the pressure by inserting a plastic cannula from a venous indwelling needle via the guidewire. Confirmation can also be performed by attaching a pressure transducer directly to the needle [91]. Do not insert a dilator unless it is certain that the guidewire resides in the vein.

- Test punctures are not recommended in small children. A hematoma caused by a test puncture will make the procedure even more difficult and could easily cause blockage.

- Even if the needle tip is definitely inside the vein, it is difficult to insert a guidewire into a vein with a small diameter; thus, consider using a guidewire with a hydrophilic monofilament. However, be careful of breakage when using metal needles.

\section{Complications}

- Owing to their small size, guidewires should not be inserted in children as deeply as in adults. Neonates and infants have thin ventricular and atrial walls that are easily penetrated.

- Cardiac tamponade is a lethal complication. The right ventricle and right atrium are common perforation sites, and perforations can occur in children from the guidewire (even J type), dilator, or catheter. Symptoms may not appear right away with a guidewire puncture, and a puncture may be difficult to diagnose with radiograph after catheterization is finished. Therefore, if a decrease in blood pressure is observed, quickly perform an echocardiogram to search for cardiac tamponade [92].

\section{Other considerations}

- Neonates have fragile skin; hence, be careful not to damage it by suturing too tightly when fixing a catheter.

Acknowledgements This practical guide was developed by the Japanese Society of Anesthesiologists' working group for safe central venous catheterization and management. The members of the group are Kimitoshi Nishiwaki, Masashi Kawamoto, Yasuyuki Shibata, Mamoru Takeuchi, Katsuya Tanaka, and Joho Tokumine. 


\section{Compliance with ethical standards}

Conflict of interest Joho Tokumine serves as a technical adviser to the Nippon Covidien Co. (Japan). Other authors have no conflict of interest.

\section{References}

1. Pikwer A, Hammarskjöld F, Larsson AT, Lindgren S, Lindwall R, Taxbro K, Oberg F, Acosta S, Akeson J. Clinical guidelines on central venous catheterisation. Swedish Society of Anaesthesiology and Intensive Care Medicine. Acta Anaesthesiol Scand. 2014;58:508-24.

2. Gallieni M, Martina V, Rizzo MA, Gravellone L, Mobilia F, Giordano A, Cusi D, Genovese U. Central venous catheters: legal issues. J Vasc Access. 2011;12:273-9.

3. Fairfax LM, Christmas AB, Norton HJ, Jacobs DG. Breakdown of the consent process at a quaternary medical center: our full disclosure. Am Surg. 2012;78:855-63.

4. Moureau N, Lamperti M, Kelly LJ, Dawson R, Elbarbary M, van Boxtel AJ, Pittiruti M. Evidence-based consensus on the insertion of central venous access devices: definition of minimal requirements for training. Br J Anaesth. 2013;110:347-56.

5. Peltan ID, Shiga T, Gordon JA, Currier PF. Simulation improves procedural protocol adherence during central venous catheter placement: a randomized controlled trial. Simul Healthc. 2015;10:270-6.

6. Hameeteman M, Bode AS, Peppelenbosch AG, van der Sande FM, Tordoir JH. Ultrasound-guided central venous catheter placement by surgical trainees: a safe procedure? J Vasc Access. 2010;11:288-92.

7. Latif RK, Bautista AF, Memon SB, Smith EA, Wang C, Wadhwa A, Carter MB, Akca O. Teaching aseptic technique for central venous access under ultrasound guidance: a randomized trial comparing didactic training alone to didactic plus simulation-based training. Anesth Analg. 2012;114:626-33.

8. Varga S, Smith J, Minneti M, Carey J, Zakaluzny S, Noguchi T, Demetriades D, Talving P. Central venous catheterization using a perfused human cadaveric model: application to surgical education. J Surg Educ. 2015;72:28-32.

9. Bannon MP, Heller SF, Rivera M. Anatomic considerations for central venous cannulation. Risk Manag Healthc Policy. 2011;4:27-39.

10. Tokumine J, Kikuchi T, Hasegawa R, Takaishi K, Sekiguchi T, Takeda Y. Practical approach for ultrasound-guided central venous catheterization (internal jugular vein, subclavian vein and femoral vein). In: Sakamoto A, Sato S, editors. Clinical anesthesia practice series 6: required skills and educational points for ultrasoundguided technique (in Japanese). Tokyo: Lifemedicom; 2013. p. $28-35$.

11. Tokumine J, Nitta K, Teruya K, Higa T, Miyata Y, Sugahara K. Ultrasound-guided internal jugular venipuncture by short-axis approach in 203 cases at the University of the Ryukyus Hospital (in Japanese with English abstract). J Jpn Soc Clin Anesth. 2008;28:439-46.

12. Tokumine J. CVC instructor's guide ver. 3 (in Japanese). Tokyo: Japanese Association for Medical Simulation; 2016. http://jams. kenkyuukai.jp/special/?id=7184.

13. Lalu MM, Fayad A, Ahmed O, Bryson GL, Fergusson DA, Barron CC, Sullivan P, Thompson C. Canadian Perioperative Anesthesia Clinical Trials Group. Ultrasound-guided subclavian vein catheterization: a systematic review and meta-analysis. Crit Care Med. 2015;43:1498-507.
14. Jiang L, Zhang M, Ma Y. Ultrasound-guided subclavian vein catheterization: a systematic review and meta-analysis: several facts need to be noticed. Crit Care Med. 2015;43:e474-5.

15. Pittiruti M, Hamilton H, Biffi R, MacFie J, Pertkiewicz M, ESPEN. ESPEN guidelines on parenteral nutrition: central venous catheters (access, care, diagnosis and therapy of complications). Clin Nutr. 2009;28:365-77.

16. Troianos CA, Hartman GS, Glas KE, Skubas NJ, Eberhardt RT, Walker JD, Reeves ST, Councils on Intraoperative Echocardiography and Vascular Ultrasound of the American Society of Echocardiography; Society of Cardiovascular Anesthesiologists. Special articles: guidelines for performing ultrasound guided vascular cannulation: recommendations of the American Society of Echocardiography and the Society of Cardiovascular Anesthesiologists. Anesth Analg. 2012;114:46-72.

17. Lamperti M, Bodenham AR, Pittiruti M, Blaivas M, Augoustides JG, Elbarbary M, Pirotte T, Karakitsos D, Ledonne J, Doniger S, Scoppettuolo G, Feller-Kopman D, Schummer W, Biffi R, Desruennes E, Melniker LA, Verghese ST. International evidence-based recommendations on ultrasound-guided vascular access. Intensive Care Med. 2012;38:1105-17.

18. Schummer W, Köditz JA, Schelenz C, Reinhart K, Sakka SG. Preprocedure ultrasound increases the success and safety of central venous catheterization. Br J Anaesth. 2014;113:122-9.

19. Shekelle PG, Dallas P. Use of real-time ultrasound guidance during central line insertion: brief update review. In: AHRQ making health care safer II. Chapter 18. http://www.ahrq.gov/research/ findings/evidence-based-reports/ptsafetyuptp.html.

20. CDC guidelines for the prevention of intravascular catheter-related infection. Atlanta: CDC; 2011. http://www.cdc.gov/hicpac/pdf/ guidelines/bsi-guidelines-2011.pdf.

21. Marik PE, Flemmer M, Harrison W. The risk of catheter-related bloodstream infection with femoral venous catheters as compared to subclavian and internal jugular venous catheters: a systematic review of the literature and meta-analysis. Crit Care Med. 2012;40:2479-85.

22. Lipshutz AK, Gropper MA. Central venous catheters: follow the evidence, not the guidelines. Crit Care Med. 2012;408:2528-9.

23. Parienti JJ, Mongardon N, Mégarbane B, Mira JP, Kalfon P, Gros A, Marqué S, Thuong M, Pottier V, Ramakers M, Savary B, Seguin A, Valette X, Terzi N, Sauneuf B, Cattoir V, Mermel LA, du Cheyron D, 3SITES Study Group. Intravascular complications of central venous catheterization by insertion site. N Engl J Med. 2015;24(373):1220-9.

24. Cotogni P, Pittiruti M. Focus on peripherally inserted central catheters in critically ill patients. World J Crit Care Med. 2014;3:80-94.

25. Mansfield PF, Hohn DC, Fornage BD, Gregurich MA, Ota DM. Complications and failures of subclavian-vein catheterization. $\mathrm{N}$ Engl J Med. 1994;331:1735-8.

26. Hatfield A, Bodenham A. Portable ultrasound for difficult central venous access. Br J Anaesth. 1999;82:822-6.

27. Brederlau J, Greim C, Schwemmer U, Haunschmid B, Markus C, Roewer N. Ultrasound-guided cannulation of the internal jugular vein in critically ill patients positioned in 30 degrees dorsal elevation. Eur J Anaesthesiol. 2004;21:684-7.

28. Tokumine J, Fujimori K. Actions for patient safety $3 \mathrm{~b}$ : safety management for high-risk procedure-guideline for central venous catheterization. In: Handbook of practicing patient safety (in Japanese). Tokyo: Japanese Coalition for Patient Safety (JCPS); 2015. p. $81-100$.

29. American Society of Anesthesiologists Task Force on Central Venous Access, Rupp SM, Apfelbaum JL, Blitt C, Caplan RA, Connis RT, Domino KB, Fleisher LA, Grant S, Mark JB, Morray JP, Nickinovich DG, Tung A. Practice guidelines for central venous access: a report by the American Society of 
Anesthesiologists Task Force on Central Venous Access. Anesthesiology. 2012;116:539-73.

30. Nishiwaki K, Ichikawa T. Actions for patient safety S: surgical safety-WHO Safe Surgery Guidelines. In: Handbook of practicing patient safety (in Japanese). Tokyo: Japanese Coalition for Patient Safety (JCPS); 2015. p. 283-306.

31. JSA monitor's guidelines for safe anesthesia (in Japanese). Tokyo: Japanese Society of Anesthesiologists; 2014. http://www.anest h.or.jp/guide/pdf/monitor3.pdf.

32. Mallory DL, Shawker T, Evans RG, McGee WT, Brenner M, Parker M. Effects of clinical maneuvers on sonographically determined internal jugular vein size during venous cannulation. Crit Care Med. 1990;18:1269-73.

33. Kawano M, Yoshimine K. Ultrasound observation of the subclavian vein: changes in the diameter with the head tilted down. J Anesth. 2007;21:448

34. Bellazzini MA, Rankin PM, Gangnon RE, Bjoernsen LP. Ultrasound validation of maneuvers to increase internal jugular vein cross-sectional area and decrease compressibility. Am J Emerg Med. 2009;27:454-9.

35. Hollenbeck KJ, Vander Schuur BM, Tulis MR, Mecklenburg BW, Gaconnet CP, Wallace SC, Lujan E, Lesnik IK. Brief report: effects of positive end-expiratory pressure on internal jugular vein cross-sectional area in anesthetized adults. Anesth Analg. 2010;110:1669-73.

36. Downey LA, Blaine KP, Sliwa J, Macario A, Brock-Utne J. Positive end-expiratory pressure to increase internal jugular vein size is poorly tolerated in obese anesthetized adults. Anesth Analg. 2014;119:619-21.

37. Stone MB, Price DD, Anderson BS. Ultrasonographic investigation of the effect of reverse trendelenburg on the cross-sectional area of the femoral vein. J Emerg Med. 2006;30:211-3.

38. Sulek CA, Gravenstein N, Blackshear RH, Weiss L. Head rotation during internal jugular vein cannulation and the risk of carotid artery puncture. Anesth Analg. 1996;82:125-8.

39. Bodenham AR, Simcock L. Complications of central venous access. In: Hamilton H, Bodenham AR, editors. Central venous catheters. Chichester: Wiley; 2009. p. 175-205.

40. Eissa NT, Kvetan V. Guide wire as a cause of complete heart block in patients with preexisting left bundle branch block. Anesthesiology. 1990;73:772-4.

41. da Silva PS, Waisberg J. Induction of life-threatening supraventricular tachycardia during central venous catheter placement: an unusual complication. J Pediatr Surg. 2010;45:E13-6.

42. Kulvatunyou N, Heard SO, Bankey PE. A subclavian artery injury, secondary to internal jugular vein cannulation, is a predictable right-sided phenomenon. Anesth Analg. 2002;95:564-6.

43. Oropello JM, Leibowitz AB, Manasia A, Del Guidice R, Benjamin E. Dilator-associated complications of central vein catheter insertion: possible mechanisms of injury and suggestions for prevention. J Cardiothorac Vasc Anesth. 1996;10:634-7.

44. Russell WC, Parker JL. Thirteen centimetre central venous catheters, lucky for all? Anaesthesia. 2003;58:388.

45. Ryu HG, Bahk JH, Kim JT, Lee JH. Bedside prediction of the central venous catheter insertion depth. Br J Anaesth. 2007;98:225-7.

46. Kim MC, Kim KS, Choi YK, Kim DS, Kwon MI, Sung JK, Moon JY, Kang JM. An estimation of right- and left-sided central venous catheter insertion depth using measurement of surface landmarks along the course of central veins. Anesth Analg. 2011;112:1371-4

47. Tokumine J. Manual of central venous catheterization using ultrasound guidance (in Japanese). Tokyo: Sogo Igaku Sha; 2007. p. 4-49.

48. Tokumine J, Lefor AT, Yonei A, Kagaya A, Iwasaki K, Fukuda Y. Three-step method for ultrasound-guided central vein catheterization. Br J Anaesth. 2013;110:368-73.
49. Stonelake PA, Bodenham AR. The carina as a radiological landmark for central venous catheter tip position. Br J Anaesth. 2006;96:335-40.

50. Domino KB, Bowdle TA, Posner KL, Spitellie PH, Lee LA, Cheney FW. Injuries and liability related to central vascular catheters: a closed claims analysis. Anesthesiology. 2004;100:1411-8.

51. Pikwer A, Acosta S, Kolbel T, Malina M, Sonesson B, Akeson J. Management of inadvertent arterial catheterization associated with central venous access procedures. Eur J Vasc Endovasc Surg. 2009;38:707-14.

52. Volpicelli G, Elbarbary M, Blaivas M, Lichtenstein DA, Mathis G, Kirkpatrick AW, Melniker L, Gargani L, Noble VE, Via G, Dean A, Tsung JW, Soldati G, Copetti R, Bouhemad B, Reissig A, Agricola E, Rouby JJ, Arbelot C, Liteplo A, Sargsyan A, Silva F, Hoppmann R, Breitkreutz R, Seibel A, Neri L, Storti E, Petrovic T, International Liaison Committee on Lung Ultrasound (ILC-LUS) for International Consensus Conference on Lung Ultrasound (ICC-LUS). International evidence-based recommendations for point-of-care lung ultrasound. Intensive Care Med. 2012:38:577-91.

53. Mirski MA, Lele AV, Fitzsimmons LF, Toung TJK. Diagnosis and treatment of vascular air embolism. Anesthesiology. 2007;106:164-77.

54. Dougherty L. How to remove a non-tunnelled central venous catheter. Nurs Stand. 2015;30:36-8.

55. Karakitsos D, Labropoulos N, De Groot E, Patrianakos AP, Kouraklis G, Poularas J, Samonis G, Tsoutsos DA, Konstadoulakis MM, Karabinis A. Real-time ultrasound-guided catheterization of the internal jugular vein: a prospective comparison with the landmark technique in critical care patients. Crit Care. 2006;10:R162. https://doi.org/10.1186/cc5101.

56. Cavanna L, Civardi G, Vallisa D, Nunzio C, Cappucciati L, Berte R, Cordani MR, Lazzaro A, Cremona G, Biasini C, Muroni M, Mordenti P, Gorgni S, Zaffignani E, Ambroggi M, Bidin L, Palladino MA, Rodino C, Tibaldi L. Ultrasound-guided central venous catheterization in cancer patients improves the success rate of cannulation and reduces mechanical complications: a prospective observational study of 1,978 consecutive catheterizations. World J Surg Oncol. 2010;8:91. https://doi.org/10.1186/1477-7819-8-91.

57. Peris A, Zagli G, Bonizzoli M, Cianchi G, Ciapetti M, Spina R, Anichini V, Lapi F, Batacchi S. Implantation of 3951 long-term central venous catheters: performances, risk analysis, and patient comfort after ultrasound-guidance introduction. Anesth Analg. 2010;111:1194-201.

58. Hind D, Calvert N, McWilliams R, Davidson A, Paisley S, Beverley $\mathrm{C}$, Thomas $\mathrm{S}$. Ultrasonic locating devices for central venous cannulation: meta-analysis. BMJ. 2003;327:361. https://doi. org/10.1136/bmj.327.7411.361.

59. Polderman KH, Girbes ARJ. Central venous catheter use. Part 1: mechanical complication. Intensive Care Med. 2002;28:1-17.

60. Hentrich M, Schalk E, Schmidt-Hieber M, Chaberny I, Mousset S, Buchheidt D, Ruhnke M, Penack O, Salwender H, Wolf HH, Christopeit M, Neumann S, Maschmeyer G, Karthaus M, Infectious Diseases Working Party of the German Society of Hematology and Medical Oncology. Central venous catheter-related infections in hematology and oncology: 2012 updated guidelines on diagnosis, management and prevention by the Infectious Diseases Working Party of the German Society of Hematology and Medical Oncology. Ann Oncol. 2014;25:936-47.

61. Schulman J, Stricof R, Stevens TP, Horgan M, Gase K, Holzman IR, Koppel RI, Nafday S, Gibbs K, Angert R, Simmonds A, Furdon SA, Saiman L, New York State Regional Perinatal Care Centers. Statewide NICU central-line-associated bloodstream infection rates decline after bundles and checklists. Pediatrics. 2011;127:436-44. 
62. Higuera F, Rosenthal VD, Duarte P, Ruiz J, Franco G, Safdar N. The effect of process control on the incidence of central venous catheter-associated bloodstream infections and mortality in intensive care units in Mexico. Crit Care Med. 2005;33:2022-7.

63. Pronovost P, Needham D, Berenholtz S, Sinopoli D, Chu H, Cosgrove S, Sexton B, Hyzy R, Welsh R, Roth G, Bander J, Kepros J, Goeschel C. An intervention to decrease catheterrelated bloodstream infections in the ICU. N Engl J Med. 2006;355:2725-32.

64. Barsuk JH, Cohen ER, Feinglass J, McGaghie WC, Wayne DB. Use of simulation-based education to reduce catheter-related bloodstream infections. Arch Intern Med. 2009;169:1420-3.

65. Spafford PS, Sinkin RA, Cox C, Reubens L, Powell KR. Prevention of central venous catheter-related coagulase-negative staphylococcal sepsis in neonates. J Pediatr. 1994;125:259-63.

66. Vassilomanolakis M, Plataniotis G, Koumakis G, Hajichristou $\mathrm{H}$, Skouteri H, Dova H, Efremidis AP. Central venous catheterrelated infections after bone marrow transplantation in patients with malignancies: a prospective study with short-course vancomycin prophylaxis. Bone Marrow Transplant. 1995;15:77-80.

67. Raad II, Hohn DC, Gilbreath BJ, Suleiman N, Hill LA, Bruso PA, Marts K, Mansfield PF, Bodey GP. Prevention of central venous catheter-related infections by using maximal sterile barrier precautions during insertion. Infect Control Hosp Epidemiol. 1994;15:231-8.

68. Miller MR, Griswold M, Harris JM 2nd, Yenokyan G, Huskins WC, Moss M, Rice TB, Ridling D, Campbell D, Margolis P, Muething S, Brilli RJ. Decreasing PICU catheter-associated bloodstream infections: NACHRI's quality transformation efforts. Pediatrics. 2010;125:206-13.

69. Berenholtz SM, Pronovost PJ, Lipsett PA, Hobson D, Earsing K, Farley JE, Milanovich S, Garrett-Mayer E, Winters BD, Rubin HR, Dorman T, Perl TM. Eliminating catheter-related bloodstream infections in the intensive care unit. Crit Care Med. 2004;32:2014-20.

70. Yasuda H, Sanui M, Japanese Society of Education for Physicians and Trainees in Intensive Care (JSEPTIC). The utility of chlorhexidine alcohol for preventing catheterrelated bloodstream infection (in Japanese with English abstract). Nichishutyuishi (J Jpn Soc Intensive Care Med). 2013;20:217-26.

71. Oda T, Hamasaki J, Kanda N, Mikami K. Anaphylactic shock induced by an antiseptic-coated central venous [correction of nervous] catheter. Anesthesiology. 1997;87:1242-4.

72. Stephens R, Mythen M, Kallis P, Davies DW, Egner W, Rickards A. Two episodes of life-threatening anaphylaxis in the same patient to a chlorhexidine-sulphadiazine-coated central venous catheter. Br J Anaesth. 2001;87:306-8.

73. Lorente L, Lecuona M, Jiménez A, Santacreu R, Raja L, Gonzalez O, Mora ML. Chlorhexidine-silver sulfadiazineimpregnated venous catheters save costs. Am J Infect Control. 2014;42:321-4.

74. Bonne S, Mazuski JE, Sona C, Schallom M, Boyle W, Buchman TG, Bochicchio GV, Coopersmith CM, Schuerer DJ. Effectiveness of minocycline and rifampin vs chlorhexidine and silver sulfadiazine-impregnated central venous catheters in preventing central line-associated bloodstream infection in a high-volume academic intensive care unit: a before and after trial. J Am Coll Surg. 2015;221:739-47.

75. Rosenthal VD, Maki DG. Prospective study of the impact of open and closed infusion systems on rates of central venous catheter-associated bacteremia. Am J Infect Control. 2004;32:135-41.

76. Rosenthal VD, Udwadia FE, Kumar S, Poojary A, Sankar R, Orellano PW, Durgad S, Thulasiraman M, Bahirune S, Kumbhar S. Clinical impact and cost-effectiveness of split-septum and single-use prefilled flushing device vs 3-way stopcock on central line-associated bloodstream infection rates in India: a randomized clinical trial conducted by the International Nosocomial Infection Control Consortium (INICC). Am J Infect Control. 2015;43:1040-5.

77. Lockman JL, Heitmiller ES, Ascenzi JA, Berkowitz I. Scrub the hub! Catheter needleless port decontamination. Anesthesiology. 2011;114:958.

78. O'Grady NP, Alexander M, Burns LA, Dellinger EP, Garland J, Heard SO, Lipsett PA, Masur H, Mermel LA, Pearson ML, Raad II, Randolph AG, Rupp ME, Saint S, Healthcare Infection Control Practices Advisory C. Guidelines for the prevention of intravascular catheter-related infections. Am J Infect Control. 2011;39:S1-34

79. Gowardman JR, Robertson IK, Parkes S, Rickard CM. Influence of insertion site on central venous catheter colonization and bloodstream infection rates. Intensive Care Med. 2008;34:1038-45.

80. Lorente L, Henry C, Martin MM, Jimenez A, Mora ML. Central venous catheter-related infection in a prospective and observational study of 2,595 catheters. Crit Care. 2005;9:R631-5.

81. McKinley S, Mackenzie A, Finfer S, Ward R, Penfold J. Incidence and predictors of central venous catheter related infection in intensive care patients. Anaesth Intensive Care. 1999;27:164-9.

82. Merrer J, De Jonghe B, Golliot F, Lefrant JY, Raffy B, Barre E, Rigaud JP, Casciani D, Misset B, Bosquet C, Outin H, BrunBuisson C, Nitenberg G, French Catheter Study Group in Intensive Care. Complications of femoral and subclavian venous catheterization in critically ill patients: a randomized controlled trial. JAMA. 2001;286:700-7.

83. Timsit JF, Schwebel C, Bouadma L, Geffroy A, GarrousteOrgeas M, Pease S, Herault MC, Haouache H, Calvino-Gunther S, Gestin B, Armand-Lefevre L, Leflon V, Chaplain C, Benali A, Francais A, Adrie C, Zahar JR, Thuong M, Arrault X, Croize J, Lucet JC, Dressing Study Group. Chlorhexidine-impregnated sponges and less frequent dressing changes for prevention of catheter-related infections in critically ill adults: a randomized controlled trial. JAMA. 2009;301:1231-41.

84. Cook D, Randolph A, Kernerman P, Cupido C, King D, Soukup C, Brun-Buisson C. Central venous catheter replacement strategies: a systematic review of the literature. Crit Care Med. 1997;25:1417-24.

85. Bonawitz SC, Hammell EJ, Kirkpatrick JR. Prevention of central venous catheter sepsis: a prospective randomized trial. Am Surg. 1991;57:618-23.

86. Kowalewska-Grochowska K, Richards R, Moysa GL, Lam K, Costerton JW, King EG. Guidewire catheter change in central venous catheter biofilm formation in a burn population. Chest. 1991;100:1090-5.

87. Casey AL, Burnell S, Whinn H, Worthington T, Faroqui MH, Elliott TS. A prospective clinical trial to evaluate the microbial barrier of a needleless connector. J Hosp Infect. 2007;65:212-8.

88. Morita M, Sasano H, Azami T, Sasano N, Fujita Y, Ito S, Sugiura T, Sobue K. A novel skin-traction method is effective for real-time ultrasound-guided internal jugular vein catheterization in infants and neonates weighing less than 5 kilograms. Anesth Analg. 2009;109:754-9.

89. Souza Neto EP, Grousson S, Duflo F, Tahon F, Mottolese C, Dailler F. Ultrasonographic anatomic variations of the major veins in paediatric patients. Br J Anaesth. 2014;112:879-84.

90. Matsushita K, Yamaura K, Karashima Y, Akiyoshi K, Hoka S. Differences in anatomical relationship between vertebral artery and internal jugular vein in children and adults measured by ultrasonography. J Clin Monit Comput. 2016;30:221-5. 
91. Oliver WC Jr, Nuttall GA, Beynen FM, Raimundo HS, Abenstein JP, Arnold JJ. The incidence of artery puncture with central venous cannulation using a modified technique for detection and prevention of arterial cannulation. J Cardiothorac Vasc Anesth. 1997;11:851-5.

92. Askegard-Giesmann JR, Caniano DA, Kenney BD. Rare but serious complications of central line insertion. Semin Pediatr Surg. 2009; 18:73-83.
Publisher's Note Springer Nature remains neutral with regard to jurisdictional claims in published maps and institutional affiliations. 